\title{
A PRIMAL-DUAL WEAK GALERKIN FINITE ELEMENT METHOD FOR FOKKER-PLANCK TYPE EQUATIONS
}

\author{
CHUNMEI WANG* AND JUNPING WANG ${ }^{\dagger}$
}

\begin{abstract}
This paper presents a primal-dual weak Galerkin (PD-WG) finite element method for a class of second order elliptic equations of Fokker-Planck type. The method is based on a variational form where all the derivatives are applied to the test functions so that no regularity is necessary for the exact solution of the model equation. The numerical scheme is designed by using locally constructed weak second order partial derivatives and the weak gradient commonly used in the weak Galerkin context. Optimal order of convergence is derived for the resulting numerical solutions. Numerical results are reported to demonstrate the performance of the numerical scheme.
\end{abstract}

Key words. primal-dual, weak Galerkin, finite element methods, Fokker-Planck equation, weak Hessian, weak gradient, polytopal partitions.

AMS subject classifications. Primary, 65N30, 65N15, 65N12, 74N20; Secondary, 35B45, 35J50, 35J35

1. Introduction. The Fokker-Planck equation plays a critical role in statistical physics and in the study of fluctuations in physical and biological systems [11, 23, 24, 27, 12. In statistical physics, it is a second order partial differential equation that describes the time evolution of the probability density function of the velocity of a particle under the influence of drag forces and random forces resulting from Gaussian white noise. The general setting of the Fokker-Planck equation is as follows. Given an open domain $\Omega \subset \mathbb{R}^{d}$ (the $d$-dimensional Euclidean space) and a terminal time $T$, we seek for a time-dependent density function $p=p(x, t): \Omega \times[0, T] \rightarrow \mathbb{R}$ satisfying

$$
\begin{aligned}
\partial_{t} p+\nabla \cdot(\boldsymbol{\mu} p)-\frac{1}{2} \sum_{i, j=1}^{d} \partial_{i j}^{2}\left(a_{i j} p\right) & =0, \quad t \in(0, T), x \in \Omega, \\
p(x, 0) & =p_{0}(x), \quad x \in \Omega,
\end{aligned}
$$

where $\partial_{i j}^{2}=\frac{\partial}{\partial x_{j}} \frac{\partial}{\partial x_{i}}$ is the second order partial derivative in the directions $x_{i}$ and $x_{j}, a(x)=\left\{a_{i j}(x)\right\}_{d \times d}$ is the diffusion tensor, $\boldsymbol{\mu}=\left(\mu_{1}, \cdots, \mu_{d}\right)$ is the drift vector, and $p_{0}=p_{0}(x)$ is the initial profile of the density function. Two common boundary conditions for 1.1 can be imposed: Dirichlet for the density function and Neumann condition for the flux. A homogeneous Dirichlet boundary data corresponds to the case where particles exit once they reach the boundary, and a prescribed flow or Neumann boundary condition represents a known current of particles crossing the boundary in the normal direction.

* Department of Mathematics, Texas State University, San Marcos, TX 78666, USA. The research of Chunmei Wang was partially supported by National Science Foundation Award DMS-1522586 and DMS-1648171.

${ }^{\dagger}$ Division of Mathematical Sciences, National Science Foundation, Arlington, VA 22230 (jwang@nsf.gov). The research of Junping Wang was supported by the NSF IR/D program, while working at National Science Foundation. However, any opinion, finding, and conclusions or recommendations expressed in this material are those of the author and do not necessarily reflect the views of the National Science Foundation. 
Numerical methods for the Fokker-Planck equation have several challenges involving various difficulties of different nature. Among them are the high dimensionality and lack of solution regularity for the probability density function. For example, in classical statistical mechanics, the Fokker-Planck equation characterizes a joint probability density function in many phase variables so that the dimension $d$ might be a big number to deal with. It can also be seen that for non-smooth diffusion tensor $a(x)$, the resulting probability density function $p=p(x)$ exhibits a shock-like discontinuity that needs to be resolved numerically. In addition, some conservation properties such as mass conservation and solution non-negativity property must be retained by the numerical solutions.

Various finite element methods have been designed for the Fokker-Planck equation for a numerical computation of the probability density function, see [3, 17, 18, 2, 26, 21, 16] and the references cited therein. In [3, 17, the stationary Fokker-Planck equation was discretized by using Galerkin finite element methods based on a weak form obtained from the usual integration by parts. In 18, another Galerkin finite element method was used to solve the Fokker-Planck equation in combination with a generalized Lagrange multiplier method to handle the associated integral constraint. In [2], the authors applied the usual $C^{0}$ finite element method to the Fokker-Planck system subject to both additive and multiplicative white noise excitations. In [21, the authors developed a framework for multi-scale finite element methods for the solution of the multi-dimensional Fokker-Planck equation in stochastic structural dynamics. All the aforementioned finite element methods assumed smooth or constant diffusion tensor $a(x)$ for the Fokker-Planck equation so that a regular weak form can be derived for the system. In [16, a $C^{0}$ finite element scheme was described and numerically tested for the transient Fokker-Planck equation without any smoothness assumption on $a(x)$; but no theory of convergence was developed for the numerical method.

For a smooth diffusion tensor, the second order differential part of the FokkerPlank equation can be reformulated as

$$
\frac{1}{2} \partial_{i j}^{2}\left(a_{i j} p\right)=\frac{1}{2} \partial_{j}\left(a_{i j}(x) \partial_{i} p\right)+\frac{1}{2} \partial_{j}\left(\left(\partial_{i} a_{i j}\right) p\right) .
$$

Therefore, the equation (1.1) can be viewed as a time-evolving convection-diffusion equation in divergence form. Aside from the high dimensionality issue, the corresponding Fokker-Planck equation is then considered as a relatively less challenging problem to solve numerically. But for non-smooth diffusion tensor, the formulation 1.2 no longer holds true, and the exact profile of the density function $p=p(x)$ possesses discontinuities that are not known a priori so that the existing finite element methods have difficulty to apply. The goal of this paper is to develop a new finite element method that addresses the numerical challenges arising from the non-smoothness nature of the diffusion tensor $a(x)$ in the Fokker-Planck equation.

For simplicity, we consider a Fokker-Planck type model equation with homogeneous Dirichlet boundary condition. The model problem seeks an unknown function $u=u(x)$ satisfying

$$
\begin{aligned}
\nabla \cdot(\boldsymbol{\mu} u)-\frac{1}{2} \sum_{i, j=1}^{d} \partial_{i j}^{2}\left(a_{i j} u\right) & =f, \quad \text { in } \Omega, \\
u & =0, \quad \text { on } \partial \Omega,
\end{aligned}
$$


where $\Omega$ is an open bounded domain in $\mathbb{R}^{d}$ with Lipschitz continuous boundary $\partial \Omega$ and $f \in L^{2}(\Omega)$ is a given function. Two of our main motivations for this selection of the model problem are: (1) a complete understanding of the Fokker-Planck equation strongly depends on the numerical properties for 1.3 as it offers a projection operator that is extremely useful in the mathematical study of $(1.1)$, and (2) the problem (1.3) itself is a poorly understood system from numerical aspects when the diffusion tensor is discontinuous. Therefore, the model problem 1.3 deserves a study as a research topic in numerical partial differential equations.

Throughout this paper, we assume that the diffusion tensor $a(x)=\left\{a_{i j}(x)\right\}_{d \times d} \in$ $\left(L^{\infty}(\Omega)\right)^{d \times d}$ is symmetric, uniformly bounded and positive definite in $\Omega$, and that the drift vector $\boldsymbol{\mu} \in\left(L^{\infty}(\Omega)\right)^{d}$. We will follow the usual notation for Sobolev spaces and norms 9 , 13, 15, 14, 4]. For any open bounded domain $D \subset \mathbb{R}^{d}$ with Lipschitz continuous boundary, we use $\|\cdot\|_{s, D}$ and $|\cdot|_{s, D}$ to denote the norm and seminorms in the Sobolev space $H^{s}(D)$ for any $s \geq 0$, respectively. The inner product in $H^{s}(D)$ is denoted by $(\cdot, \cdot)_{s, D}$. The space $H^{0}(D)$ coincides with $L^{2}(D)$, for which the norm and the inner product are denoted by $\|\cdot\|_{D}$ and $(\cdot, \cdot)_{D}$, respectively. When $D=\Omega$, we shall drop the subscript $D$ in the norm and inner product notation. For convenience, we use " $\lesssim$ " to denote "less than or equal to up to a general constant independent of the mesh size or functions appearing in the inequality".

By a weak solution of 1.3 we mean a function $u=u(x) \in L^{2}(\Omega)$ satisfying

$$
(u, \mathcal{L} v)=-(f, v), \quad \forall v \in H^{2}(\Omega) \cap H_{0}^{1}(\Omega),
$$

where $\mathcal{L}$ is a differential operator given by $\mathcal{L} v=\boldsymbol{\mu} \cdot \nabla v+\frac{1}{2} \sum_{i, j=1}^{d} a_{i j} \partial_{j i}^{2} v$. The differential operator $\mathcal{L}$ is assumed to satisfy the $H^{2}$-regularity property in the sense that for any given $\chi \in L^{2}(\Omega)$, there exists a unique strong solution $\Phi \in H^{2}(\Omega) \cap H_{0}^{1}(\Omega)$ satisfying

$$
\mathcal{L} \Phi=\chi, \quad\|\Phi\|_{2} \lesssim\|\chi\| .
$$

In 25, it was shown that the regularity assumption 1.5 holds true on bounded convex domain $\Omega$ if $\boldsymbol{\mu}=0$ and the diffusion tensor satisfies the following Cordès condition

$$
\frac{\sum_{i, j=1}^{d} a_{i j}^{2}}{\left(\sum_{i=1}^{d} a_{i i}\right)^{2}} \leq \frac{1}{d-1+\varepsilon} \quad \text { in } \Omega,
$$

for a parameter $\varepsilon \in(0,1]$. The Cordès condition 1.6$)$ is automatically satisfied in $2 \mathrm{D}$ for diffusion tensor that is bounded, symmetric, and uniformly positive definite in the domain, see [30] for a verification.

Our numerical scheme for the model problem 1.3 is based on the weak formulation (1.4) through a weak Galerkin approach that combines the primal variable with its dual. The dual problem for the primal equation $(1.4)$ is given by

$$
(w, \mathcal{L} \rho)=0, \quad \forall w \in L^{2}(\Omega),
$$

where $\rho \in H^{2}(\Omega) \cap H_{0}^{1}(\Omega)$ is the dual variable. Under the $H^{2}$-regularity assumption 1.5), the solution to the dual problem (1.7) is clearly trivial; i.e., $\rho \equiv 0$. Note that the primal and the dual equations are formally uncorrelated to each other in 
the continuous case; but this changes significantly in the context of weak Galerkin finite element methods. In the weak Galerkin approach, the differential operator $\mathcal{L}$ is discretized as

$$
\mathcal{L}_{w}(v):=\boldsymbol{\mu} \cdot \nabla_{w} v+\frac{1}{2} \sum_{i, j=1}^{d} a_{i j} \partial_{j i, w}^{2} v,
$$

where $\nabla_{w}$ is a discrete weak gradient [31, 19, 33, 32] and $\partial_{j i, w}^{2}$ is a discrete weak second order partial derivative [20, 28, (also see Section 2 for their definition). The corresponding primal and dual equation then become to be

$$
\text { discrete primal: } \quad\left(u_{h}, \mathcal{L}_{w} v\right)=-(f, v), \quad \forall v \in V_{h, k}^{0}
$$

and

$$
\text { discrete dual: }\left(w, \mathcal{L}_{w} \rho_{h}\right)=0, \quad \forall w \in W_{h, s},
$$

where $V_{h, k}^{0}$ and $W_{h, s}$ are two weak finite element spaces used to approximate $H^{2}(\Omega) \cap$ $H_{0}^{1}(\Omega)$ and $L^{2}(\Omega)$ respectively. While neither 1.8 nor 1.9 makes any computationally feasible schemes, their combination through the use of a suitably-defined stabilizer does provide numerical methods that are efficient, accurate, and stable for several model problems $[7,6,6,30$. A formal description of the scheme reads as follows: Find $u_{h} \in W_{h, s}$ and $\rho_{h} \in V_{h, k}^{0}$ such that

$$
\begin{aligned}
s\left(\rho_{h}, v\right)+\left(u_{h}, \mathcal{L}_{w} v\right) & =-(f, v), \quad \forall v \in V_{h, k}^{0}, \\
\left(w, \mathcal{L}_{w} \rho_{h}\right) & =0, \quad \forall w \in W_{h, s},
\end{aligned}
$$

where $s(\cdot, \cdot)$ is a bilinear form in $V_{h, k}^{0} \times V_{h, k}^{0}$ known as stabilizer or smoother that enforces a certain weak continuity for the approximation $\rho_{h}$. Numerical schemes in the form of 1.10 were named primal-dual weak Galerkin finite element methods in [30, but were broadly called stabilized finite element methods in [7, 8, 6].

In the rest of the paper, we will provide all the technical details for the numerical scheme 1.10, including the construction of the finite element spaces $V_{h, k}^{0}$ and $W_{h, s}$, representation of the stabilizer or smoother $s(\cdot, \cdot)$, mathematical convergence for the corresponding numerical approximations, and some numerical results that demonstrate the performance of the method. One of the distinguished features of this approach lies on ultra weak regularity assumptions for the primal variable $u=u(x)$ in the mathematical convergence theory. The method essentially assumes no regularity on the primal variable so that solutions with discontinuity can be well approximated by our primal-dual finite element method. This work is a non-trivial extension of 30] in both theory and algorithmic development.

The paper is organized as follows. In Section 2 , we shall briefly discuss the computation of weak gradients and weak second order partial derivatives. In Section 3. we will present a detailed description of the primal-dual weak Galerkin finite element method for the Fokker-Planck type model problem 1.3 based on the weak formulation (1.4). In Section 4, we will study the solution properties for our numerical method. In particular, we shall derive an inf-sup condition, and then establish a result on the solution existence and uniqueness. In Section 5 , we will derive an error equation for the numerical solutions. Then in Section 6, we will establish an error 
estimate for the primal variable that is of optimal order in $L^{2}$. Section 7 is devoted to a presentation of error estimates for the usual $L^{2}$ projections. Finally in Section 8 , we report some numerical results to demonstrate the performance of the primal-dual weak Galerkin finite element method.

2. Weak Partial Derivatives. The goal of this section is to brief the definition and computation of the discrete weak partial derivatives introduced in [28, 29, 32]. To this end, let $T$ be a polygonal or polyhedral region with boundary $\partial T$. By a weak function on $T$ we mean a triplet $v=\left\{v_{0}, v_{b}, \mathbf{v}_{g}\right\}$ in which $v_{0} \in L^{2}(T), v_{b} \in L^{2}(\partial T)$ and $\mathbf{v}_{g} \in\left[L^{2}(\partial T)\right]^{d}$. The first and second components $v_{0}$ and $v_{b}$ are intended for the value of $v$ in the interior and on the boundary of $T$, respectively. The third component $\mathbf{v}_{g}=\left(v_{g 1}, \cdots, v_{g d}\right) \in \mathbb{R}^{d}$ is used to represent the gradient of $v$ on $\partial T$. In general, $v_{b}$ and $\mathbf{v}_{g}$ are not required to be consistent with the trace of $v_{0}$ and $\nabla v_{0}$ on $\partial T$. Denote by $\mathcal{W}(T)$ the space of all weak functions on $T$ :

$$
\mathcal{W}(T)=\left\{v=\left\{v_{0}, v_{b}, \mathbf{v}_{g}\right\}: v_{0} \in L^{2}(T), v_{b} \in L^{2}(\partial T), \mathbf{v}_{g} \in\left[L^{2}(\partial T)\right]^{d}\right\} .
$$

For any $v \in \mathcal{W}(T)$, the weak second order partial derivative $\partial_{i j}^{2} v$, denoted as $\partial_{i j, w}^{2} v$, is defined as a linear functional in the dual space of $H^{2}(T)$ satisfying

$$
\left(\left(\partial_{i j, w}^{2} v, \varphi\right)\right)_{T}=\left(v_{0}, \partial_{j i}^{2} \varphi\right)_{T}-\left\langle v_{b} n_{i}, \partial_{j} \varphi\right\rangle_{\partial T}+\left\langle v_{g i}, \varphi n_{j}\right\rangle_{\partial T},
$$

for all $\varphi \in H^{2}(T)$. Here, $((\chi, \varphi))_{T}$ stands for the action of $\chi$ at $\varphi \in H^{2}(T), \mathbf{n}=$ $\left(n_{1}, \cdots, n_{d}\right)$ is the unit outward normal direction to $\partial T,(\cdot, \cdot)_{T}$ is the usual $L^{2}$ inner product in $L^{2}(T)$, and $\langle\cdot, \cdot\rangle_{\partial T}$ is the $L^{2}$ inner product in $L^{2}(\partial T)$.

The weak gradient of $v \in \mathcal{W}(T)$, denoted by $\nabla_{w} v$, is defined as a linear functional in the dual space of $\left[H^{1}(T)\right]^{d}$ such that

$$
\left(\left(\nabla_{w} v, \boldsymbol{\psi}\right)\right)_{T}=-\left(v_{0}, \nabla \cdot \boldsymbol{\psi}\right)_{T}+\left\langle v_{b}, \boldsymbol{\psi} \cdot \mathbf{n}\right\rangle_{\partial T},
$$

for all $\boldsymbol{\psi} \in\left[H^{1}(T)\right]^{d}$. Note that the weak gradient makes no use of the third component of the weak function $v$.

Denote by $P_{r}(T)$ the set of polynomials on $T$ with degree no more than $r$. A discrete version of $\partial_{i j, w}^{2} v$ for $v \in \mathcal{W}(T)$, denoted by $\partial_{i j, w, r, T}^{2} v$, is defined as the unique polynomial in $P_{r}(T)$ satisfying

$$
\left(\partial_{i j, w, r, T}^{2} v, \varphi\right)_{T}=\left(v_{0}, \partial_{j i}^{2} \varphi\right)_{T}-\left\langle v_{b} n_{i}, \partial_{j} \varphi\right\rangle_{\partial T}+\left\langle v_{g i}, \varphi n_{j}\right\rangle_{\partial T},
$$

for all $\varphi \in P_{r}(T)$, which, by using integration by parts, yields

$$
\left(\partial_{i j, w, r, T}^{2} v, \varphi\right)_{T}=\left(\partial_{i j}^{2} v_{0}, \varphi\right)_{T}+\left\langle\left(v_{0}-v_{b}\right) n_{i}, \partial_{j} \varphi\right\rangle_{\partial T}-\left\langle\partial_{i} v_{0}-v_{g i}, \varphi n_{j}\right\rangle_{\partial T},
$$

for all $\varphi \in P_{r}(T)$.

A discrete form of $\nabla_{w} v$ for $v \in \mathcal{W}(T)$, denoted by $\nabla_{w, r, T} v$, is defined as the unique polynomial vector in $\left[P_{r}(T)\right]^{d}$ satisfying

$$
\left(\nabla_{w, r, T} v, \boldsymbol{\psi}\right)_{T}=-\left(v_{0}, \nabla \cdot \boldsymbol{\psi}\right)_{T}+\left\langle v_{b}, \boldsymbol{\psi} \cdot \mathbf{n}\right\rangle_{\partial T}, \quad \forall \boldsymbol{\psi} \in\left[P_{r}(T)\right]^{d},
$$

which, from integration by parts, gives

$$
\left(\nabla_{w, r, T} v, \boldsymbol{\psi}\right)_{T}=\left(\nabla v_{0}, \boldsymbol{\psi}\right)_{T}-\left\langle v_{0}-v_{b}, \boldsymbol{\psi} \cdot \mathbf{n}\right\rangle_{\partial T}, \quad \forall \boldsymbol{\psi} \in\left[P_{r}(T)\right]^{d},
$$

provided that $v_{0}$ is sufficiently regular. 
3. Numerical Schemes. The goal of this section is to present a finite element method for the variational problem (1.4). To this end, let $\mathcal{T}_{h}$ be a finite element partition of the domain $\Omega$ into polygons in $2 \mathrm{D}$ or polyhedra in $3 \mathrm{D}$ which is shape regular in the sense as described in [32. For three dimensional domains, all the polyhedral elements are assumed to have flat faces. Denote by $\mathcal{E}_{h}$ the set of all edges or faces in $\mathcal{T}_{h}$ and $\mathcal{E}_{h}^{0}=\mathcal{E}_{h} \backslash \partial \Omega$ the set of all interior edges or faces. Denote by $h_{T}$ the meshsize of $T \in \mathcal{T}_{h}$ and $h=\max _{T \in \mathcal{T}_{h}} h_{T}$ the meshsize of the partition $\mathcal{T}_{h}$.

Let $k \geq 1$ be a given integer. Denote by $V_{k}(T)$ the discrete local weak function space on $T$ given by

$$
V_{k}(T)=\left\{\left\{v_{0}, v_{b}, \mathbf{v}_{g}\right\}: v_{0} \in P_{k}(T), v_{b} \in P_{k}(e), \mathbf{v}_{g} \in\left[P_{k-1}(e)\right]^{d}, e \subset \partial T\right\} .
$$

By patching $V_{k}(T)$ over all the elements $T \in \mathcal{T}_{h}$ through a common value $v_{b}$ and $\mathbf{v}_{g}$ on the interior edges/faces, we obtain a global weak finite element space $V_{h, k}$ :

$$
V_{h, k}=\left\{\left\{v_{0}, v_{b}, \mathbf{v}_{g}\right\}:\left.\quad\left\{v_{0}, v_{b}, \mathbf{v}_{g}\right\}\right|_{T} \in V_{k}(T), T \in \mathcal{T}_{h}\right\} .
$$

Denote by $V_{h, k}^{0}$ the subspace of $V_{h, k}$ with vanishing boundary value for $v_{b}$ on $\partial \Omega$, i.e.,

$$
V_{h, k}^{0}=\left\{\left\{v_{0}, v_{b}, \mathbf{v}_{g}\right\} \in V_{h, k}:\left.\quad v_{b}\right|_{e}=0, e \subset \partial \Omega\right\} .
$$

For any given integer $s \geq 0$, denote by $W_{h, s}$ the usual finite element space consisting of piecewise polynomials of degree $s$; i.e.,

$$
W_{h, s}=\left\{w:\left.\quad w\right|_{T} \in P_{s}(T), T \in \mathcal{T}_{h}\right\} .
$$

For application in the approximation of (1.4), the integer $s$ will be chosen as either $s=k-1$ or $s=k-2$. In the case of $k=1$, the only viable option for this integer would be $s=0$.

For simplicity of notation, denote by $\nabla_{w} \sigma$ the discrete weak gradient $\nabla_{w, k-1, T} \sigma$ computed by using 2.5) on each element $T$ with $r=k-1$ :

$$
\left.\left(\nabla_{w} \sigma\right)\right|_{T}=\nabla_{w, k-1, T}\left(\left.\sigma\right|_{T}\right), \quad \sigma \in V_{h, k} .
$$

Analogously, we use $\partial_{i j, w}^{2} \sigma$ to denote the discrete weak second order partial derivative $\partial_{i j, w, s, T}^{2} \sigma$ computed by using 2.3 on each element $T$ with $r=s$ :

$$
\left.\left(\partial_{i j, w}^{2} \sigma\right)\right|_{T}=\partial_{i j, w, s, T}^{2}\left(\left.\sigma\right|_{T}\right), \quad \sigma \in V_{h, k} .
$$

The corresponding weak differential operator is defined by using weak partial derivatives as follows

$$
\mathcal{L}_{w}(\sigma)=\mu \cdot \nabla_{w} \sigma+\frac{1}{2} \sum_{i, j=1}^{d} a_{i j} \partial_{j i, w}^{2} \sigma
$$

for any $\sigma \in V_{h, k}$.

Let us introduce the following bilinear forms

$$
\begin{array}{ll}
s(\rho, \sigma)=\sum_{T \in \mathcal{T}_{h}} s_{T}(\rho, \sigma), & \rho, \sigma \in V_{h, k}, \\
b(\sigma, v)=\sum_{T \in \mathcal{T}_{h}} b_{T}(\sigma, v), & \sigma \in V_{h, k}, v \in W_{h, s},
\end{array}
$$


where

$$
\begin{aligned}
s_{T}(\rho, \sigma)= & h_{T}^{-3} \int_{\partial T}\left(\rho_{0}-\rho_{b}\right)\left(\sigma_{0}-\sigma_{b}\right) d s \\
& +h_{T}^{-1} \int_{\partial T}\left(\nabla \rho_{0}-\boldsymbol{\rho}_{g}\right)\left(\nabla \sigma_{0}-\boldsymbol{\sigma}_{g}\right) d s \\
& +\delta \int_{T} \mathcal{L}\left(\rho_{0}\right) \mathcal{L}\left(\sigma_{0}\right) d T
\end{aligned}
$$

and

$$
b_{T}(\sigma, v)=\left(v, \mathcal{L}_{w}(\sigma)\right)_{T} .
$$

Here, $\delta>0$ is a parameter independent of the meshsize $h$ and the functions involved.

We are now in a position to state our primal-dual weak Galerkin finite element scheme for the model variational problem (1.4).

Primal-Dual Weak Galerkin Algorithm 3.1. Let $k \geq 1$ be a given integer and $s \geq 0$ be another integer. A numerical approximation for the solution of (1.4) is the component $u_{h}$ in $\left(u_{h} ; \rho_{h}\right) \in W_{h, s} \times V_{h, k}^{0}$ satisfying

$$
\begin{aligned}
s\left(\rho_{h}, \sigma\right)+b\left(\sigma, u_{h}\right) & =-\left(f, \sigma_{0}\right), & & \forall \sigma \in V_{h, k}^{0}, \\
b\left(\rho_{h}, v\right) & =0, & & \forall v \in W_{h, s} .
\end{aligned}
$$

4. Solution Existence, Uniqueness, and Stability. The goal of this section is to study the solution for the numerical scheme (3.2)-(3.3). In particular, we shall prove the existence and uniqueness for the numerical solution under certain assumptions on the finite element partition $\mathcal{T}_{h}$ and the differential operator $\mathcal{L}$.

On each element $T \in \mathcal{T}_{h}$, denote by $Q_{0}$ the $L^{2}$ projection onto $P_{k}(T), k \geq 1$. Similarly, on each edge or face $e \subset \partial T$, denote by $Q_{b}$ and $\mathbf{Q}_{g}:=\left(Q_{g 1}, \cdots, Q_{g d}\right)$ the $L^{2}$ projections onto $P_{k}(e)$ and $\left[P_{k-1}(e)\right]^{d}$, respectively. For any $w \in H^{2}(\Omega)$, define the projection $Q_{h} w \in V_{h, k}$ so that on each element $T$ one has

$$
Q_{h} w=\left\{Q_{0} w, Q_{b} w, \mathbf{Q}_{g}(\nabla w)\right\}
$$

Denote by $\mathcal{Q}_{h}^{(s)}$ the $L^{2}$ projection onto $W_{h, s}$ - the space of piecewise polynomials of degree $s \geq 0$. In the rest of this paper, the integer $s$ will be taken as either $s=k-1$ or $s=k-2$.

LEMma 4.1. [28, 29, 32] The aforementioned projection operators satisfy the following commutative properties: For any $w \in H^{2}(T)$, one has

$$
\begin{aligned}
\partial_{i j, w}^{2}\left(Q_{h} w\right) & =\mathcal{Q}_{h}^{(s)}\left(\partial_{i j}^{2} w\right), \quad i, j=1, \ldots, d, \\
\nabla_{w}\left(Q_{h} w\right) & =\mathcal{Q}_{h}^{(k-1)}(\nabla w) .
\end{aligned}
$$


Proof. For any $\varphi \in P_{s}(T)$ and $w \in H^{2}(T)$, from 2.3 , the usual property of $L^{2}$ projections, and the integration by parts, we have

$$
\begin{aligned}
\left(\partial_{i j, w}^{2}\left(Q_{h} w\right), \varphi\right)_{T} & =\left(Q_{0} w, \partial_{j i}^{2} \varphi\right)_{T}-\left\langle Q_{b} w, \partial_{j} \varphi \cdot n_{i}\right\rangle_{\partial T}+\left\langle Q_{g i}\left(\partial_{i} w\right) \cdot n_{j}, \varphi\right\rangle_{\partial T} \\
& =\left(w, \partial_{j i}^{2} \varphi\right)_{T}-\left\langle w, \partial_{j} \varphi \cdot n_{i}\right\rangle_{\partial T}+\left\langle\partial_{i} w \cdot n_{j}, \varphi\right\rangle_{\partial T} \\
& =\left(\partial_{i j}^{2} w, \varphi\right)_{T} \\
& =\left(\mathcal{Q}_{h}^{(s)} \partial_{i j}^{2} w, \varphi\right)_{T}
\end{aligned}
$$

which completes the proof of 4.2 . The other identity 4.3 can be derived in a similar fashion, and details can be found in [28, 29, 32].

The stabilizer $s(\cdot, \cdot)$ defined through (3.1) naturally induces a semi-norm in the weak finite element space $V_{h, k}$ as follows:

$$
\|\rho\|_{w}=s(\rho, \rho)^{\frac{1}{2}}, \quad \rho \in V_{h, k} .
$$

LEMмA 4.2. (inf-sup condition) Assume that the drift term $\mu \in L^{\infty}(\Omega)$ and the coefficient tensor $a(x)$ is uniformly piecewise continuous with respect to the finite element partition $\mathcal{T}_{h}$. Then, there exists a constant $\beta>0$ such that for any $v \in W_{h, s}$, there exists a weak function $\sigma \in V_{h, k}^{0}$ satisfying

$$
\begin{aligned}
b(v, \sigma) & \geq \frac{1}{2}\|v\|^{2}, \\
\|\sigma\|_{w} & \leq \beta\|v\|,
\end{aligned}
$$

provided that meshsize satisfies $h \leq h_{0}$ for a small, but fixed parameter value $h_{0}>0$.

Proof. Let $\Phi$ be the solution of the following auxiliary problem:

$$
\begin{aligned}
\mathcal{L} \Phi & =v, & & \text { in } \Omega, \\
\Phi & =0, & & \text { on } \partial \Omega .
\end{aligned}
$$

From the assumption 1.5 , the problem 4.7 - 4.8 has the following $H^{2}$-regularity estimate

$$
\|\Phi\|_{2} \leq C\|v\|
$$


With $\sigma=Q_{h} \Phi$, we have from Lemma 4.1 that

$$
\begin{aligned}
b(v, \sigma)= & \sum_{T \in \mathcal{T}_{h}}\left(v, \mathcal{L}_{w}\left(Q_{h} \Phi\right)\right)_{T} \\
= & \sum_{T \in \mathcal{T}_{h}}\left(\boldsymbol{\mu} v, \nabla_{w} Q_{h} \Phi\right)_{T}+\frac{1}{2} \sum_{i, j=1}^{d}\left(a_{i j} v, \partial_{j i, w}^{2} Q_{h} \Phi\right)_{T} \\
= & \sum_{T \in \mathcal{T}_{h}}\left(\boldsymbol{\mu} v, \mathcal{Q}_{h}^{(k-1)}(\nabla \Phi)\right)_{T}+\frac{1}{2} \sum_{i, j=1}^{d}\left(a_{i j} v, \mathcal{Q}_{h}^{(s)} \partial_{j i}^{2} \Phi\right)_{T} \\
= & \sum_{T \in \mathcal{T}_{h}}(\boldsymbol{\mu} v, \nabla \Phi)_{T}+\frac{1}{2} \sum_{i, j=1}^{d}\left(a_{i j} v, \partial_{j i}^{2} \Phi\right)_{T} \\
& +\left(\boldsymbol{\mu} v,\left(\mathcal{Q}_{h}^{(k-1)}-I\right) \nabla \Phi\right)_{T}+\frac{1}{2} \sum_{i, j=1}^{d}\left(a_{i j} v,\left(\mathcal{Q}_{h}^{(s)}-I\right) \partial_{j i}^{2} \Phi\right)_{T} \\
= & \sum_{T \in \mathcal{T}_{h}}(v, v)_{T}+\sum_{T \in \mathcal{T}_{h}}\left(\boldsymbol{\mu} v,\left(\mathcal{Q}_{h}^{(k-1)}-I\right) \nabla \Phi\right)_{T} \\
& +\frac{1}{2} \sum_{i, j=1}^{d}\left(\left(a_{i j}-\bar{a}_{i j}\right) v,\left(\mathcal{Q}_{h}^{(s)}-I\right) \partial_{i j}^{2} \Phi\right)_{T},
\end{aligned}
$$

where $\bar{a}_{i j}$ stands for the average of $a_{i j}$ on each element $T \in \mathcal{T}_{h}$. As the coefficient tensor $a(x)=\left(a_{i j}(x)\right)_{d \times d}$ is uniformly piecewise continuous in $\Omega$, there exists a small parameter $\varepsilon(h)$ depending on the meshsize $h$ and the continuity of $a_{i j}$ on each element $T$ such that

$$
\begin{aligned}
& \left|\sum_{T \in \mathcal{T}_{h}}\left(\boldsymbol{\mu} v,\left(\mathcal{Q}_{h}^{(k-1)}-I\right) \nabla \Phi\right)_{T}\right| \leq C h\|\Phi\|_{2}\|v\| \\
& \left|\sum_{i, j=1}^{d}\left(\left(a_{i j}-\bar{a}_{i j}\right) v,\left(\mathcal{Q}_{h}^{(s)}-I\right) \partial_{i j}^{2} \Phi\right)_{T}\right| \leq C \varepsilon(h)\|\Phi\|_{2}\|v\| .
\end{aligned}
$$

Substituting the above estimates into 4.10 yields

$$
\begin{aligned}
b(v, \sigma) & \geq\|v\|^{2}-C(h+\varepsilon(h))\|\Phi\|_{2}\|v\| \\
& \geq(1-C h-C \varepsilon(h))\|v\|^{2},
\end{aligned}
$$

where we have used the regularity estimate 4.9 . In particular, for $\varepsilon_{0}=\frac{1}{2 C}$, there exists a parameter value $h_{0}$ such that $h+\varepsilon(h) \leq \varepsilon_{0}$ when $h \leq h_{0}$. It follows that $(h+\varepsilon(h)) C \leq \frac{1}{2}$ holds true for $h \leq h_{0}$, and hence

$$
b(v, \sigma) \geq \frac{1}{2}\|v\|^{2},
$$

which verifies the inequality 4.5 .

It remains to establish the estimate (4.6) for $\sigma=Q_{h} \Phi$. To this end, from the 
usual trace inequality we have

$$
\begin{aligned}
& \sum_{T \in \mathcal{T}_{h}} h_{T}^{-3} \int_{\partial T}\left|\sigma_{0}-\sigma_{b}\right|^{2} d s \\
= & \sum_{T \in \mathcal{T}_{h}} h_{T}^{-3} \int_{\partial T}\left|Q_{0} \Phi-Q_{b} \Phi\right|^{2} d s \\
\leq & \sum_{T \in \mathcal{T}_{h}} h_{T}^{-3} \int_{\partial T}\left|Q_{0} \Phi-\Phi\right|^{2} d s \\
\lesssim & \sum_{T \in \mathcal{T}_{h}} h_{T}^{-4} \int_{T}\left|Q_{0} \Phi-\Phi\right|^{2} d T+h_{T}^{-2} \int_{T}\left|\nabla Q_{0} \Phi-\nabla \Phi\right|^{2} d T \\
\lesssim & \|\Phi\|_{2}^{2} \lesssim\|v\|^{2} .
\end{aligned}
$$

A similar analysis can be applied to yield the following estimate:

$$
\sum_{T \in \mathcal{T}_{h}} h_{T}^{-1} \int_{\partial T}\left|\nabla \sigma_{0}-\sigma_{g}\right|^{2} d s \lesssim\|v\|^{2} .
$$

Furthermore, we have

$$
\begin{aligned}
\sum_{T \in \mathcal{T}_{h}} \delta \int_{T}\left|\mathcal{L}\left(\sigma_{0}\right)\right|^{2} d T & \lesssim \sum_{T \in \mathcal{T}_{h}}\left\|\sigma_{0}\right\|_{2, T}^{2} \\
& \lesssim \sum_{T \in \mathcal{T}_{h}}\left\|Q_{0} \Phi\right\|_{2, T}^{2} \\
& \lesssim\|\Phi\|_{2}^{2} \lesssim\|v\|^{2} .
\end{aligned}
$$

Finally, by combining the estimates 4.13)-4.15 and the definition of $\|\sigma\|_{w}$ we obtain

$$
\|\sigma\|_{w}^{2} \leq \beta^{2}\|v\|^{2}
$$

for some constant $\beta$. This completes the proof of the lemma.

We are now in a position to state the main result on solution existence and uniqueness.

THEOREM 4.3. Assume that the drift vector $\boldsymbol{\mu}$ and the coefficient tensor a $(x)$ are uniformly piecewise continuous in $\Omega$ with respect to the finite element partition $\mathcal{T}_{h}$. Then, there exists a fixed $h_{0}>0$ such that the primal-dual weak Galerkin finite element algorithm (3.2)-(3.3) has one and only one solution if the meshsize satisfies $h \leq h_{0}$.

Proof. It suffices to show that zero is the only solution to the problem $3.2 \mathrm{~s}-(3.3)$ with homogeneous data $f=0$ and $g=0$. To this end, assume $f=0$ and $g=0$ in 3.2 -3.3. By choosing $v=u_{h}$ and $\sigma=\rho_{h}$, the difference of 3.3) and 3.2 gives $s\left(\rho_{h}, \rho_{h}\right)=0$, which implies $\rho_{0}=\rho_{b}$ and $\nabla \rho_{0}=\rho_{g}$ on each $\partial T$, and hence $\rho_{0} \in C_{0}^{1}(\Omega)$. Moreover, we have

$$
\mathcal{L} \rho_{0}=0 .
$$

Thus, from the solution existence and uniqueness for the differential operator $\mathcal{L}$ with homogeneous Dirichlet boundary condition we obtain $\rho_{0} \equiv 0$, and hence $\rho_{h} \equiv 0$. 
With $\rho_{h}=0$, the equation 3.2 now becomes

$$
b\left(u_{h}, \tau\right)=0, \quad \forall \tau \in V_{h, k}^{0} .
$$

From Lemma 4.2 there exists a $\sigma \in V_{h, k}^{0}$ such that $b\left(u_{h}, \sigma\right) \geq \frac{1}{2}\left\|u_{h}\right\|^{2}$. It then follows from 4.16 that $u_{h} \equiv 0$. This completes the proof of the theorem. $\square$

5. Error Equations. In this section we shall derive an error equation for the numerical solution arising from the primal-dual weak Galerkin finite element algorithm (3.2)-(3.3). To this end, let $u$ and $\left(u_{h} ; \rho_{h}\right) \in W_{h, s} \times V_{h, k}^{0}$ be the solution of (1.4) and (3.2)-(3.3), respectively. Note that $\rho_{h}$ is supposed to approximate the trivial function $\rho=0$.

Lemma 5.1. For any $\sigma \in V_{h, k}$ and $v \in W_{h, s}$, the following identity holds true:

$$
\left(\mathcal{L}_{w} \sigma, v\right)_{T}=\left(\mathcal{L} \sigma_{0}, v\right)_{T}+R_{T}(\sigma, v)
$$

where

$$
\begin{aligned}
R_{T}(\sigma, v)= & \frac{1}{2} \sum_{i, j=1}^{d}\left\langle\sigma_{0}-\sigma_{b}, n_{j} \partial_{i}\left(\mathcal{Q}_{h}^{(s)}\left(a_{i j} v\right)\right)\right\rangle_{\partial T} \\
& -\frac{1}{2} \sum_{i, j=1}^{d}\left\langle\partial_{j} \sigma_{0}-\sigma_{g j}, n_{i} \mathcal{Q}_{h}^{(s)}\left(a_{i j} v\right)\right\rangle_{\partial T} \\
& -\left\langle\sigma_{0}-\sigma_{b}, \mathcal{Q}_{h}^{(k-1)}(\boldsymbol{\mu} v) \cdot \mathbf{n}\right\rangle_{\partial T} .
\end{aligned}
$$

Proof. From the formula (2.4) and (2.6) for the weak derivatives, we have

$$
\begin{aligned}
& \left(\mathcal{L}_{w}(\sigma), v\right)_{T} \\
= & \left(\boldsymbol{\mu} \cdot \nabla_{w} \sigma, v\right)_{T}+\frac{1}{2} \sum_{i, j=1}^{d}\left(a_{i j} \partial_{j i, w}^{2} \sigma, v\right)_{T} \\
= & \left(\nabla_{w} \sigma, \mathcal{Q}_{h}^{(k-1)}(\boldsymbol{\mu} v)\right)_{T}+\frac{1}{2} \sum_{i, j=1}^{d}\left(\partial_{j i, w}^{2} \sigma, \mathcal{Q}_{h}^{(s)}\left(a_{i j} v\right)\right)_{T} \\
= & \left(\nabla \sigma_{0}, \boldsymbol{\mu} v\right)_{T}+\frac{1}{2} \sum_{i, j=1}^{d}\left(\partial_{j i}^{2} \sigma_{0}, a_{i j} v\right)_{T}+R_{T}(\sigma, v) \\
= & \left(\mathcal{L} \sigma_{0}, v\right)_{T}+R_{T}(\sigma, v),
\end{aligned}
$$

where $R_{T}(\sigma, v)$ is given by $(5.2)$.

By error functions we mean the difference between the numerical solution and the $L^{2}$ interpolation of the exact solution; namely,

$$
\begin{aligned}
& e_{h}=u_{h}-\mathcal{Q}_{h}^{(s)} u, \\
& \epsilon_{h}=\rho_{h}-Q_{h} \rho=\rho_{h} .
\end{aligned}
$$

LEMma 5.2. Let $u$ and $\left(u_{h} ; \rho_{h}\right) \in W_{h, s} \times V_{h, k}^{0}$ be the solutions arising from (1.3) and (3.2)-(3.3), respectively. Then, the error functions $e_{h}$ and $\epsilon_{h}$ satisfy the following 
equations

$$
\begin{aligned}
s\left(\epsilon_{h}, \sigma\right)+b\left(\sigma, e_{h}\right) & =\ell_{u}(\sigma), & & \forall \sigma \in V_{h, k}^{0}, \\
b\left(\epsilon_{h}, v\right) & =0, & & \forall v \in W_{h, s},
\end{aligned}
$$

where $\ell_{u}(\sigma)$ is given by

$$
\begin{aligned}
\ell_{u}(\sigma)= & \sum_{T \in \mathcal{T}_{h}}\left\langle\sigma_{b}-\sigma_{0},\left(\boldsymbol{\mu} u-\mathcal{Q}_{h}^{(k-1)}\left(\boldsymbol{\mu} \mathcal{Q}_{h}^{(s)} u\right)\right) \cdot \mathbf{n}\right\rangle_{\partial T} \\
& +\frac{1}{2} \sum_{T \in \mathcal{T}_{h}} \sum_{i, j=1}^{d}\left\langle\sigma_{0}-\sigma_{b}, n_{j} \partial_{i}\left(a_{i j} u-\mathcal{Q}_{h}^{(s)}\left(a_{i j} \mathcal{Q}_{h}^{(s)} u\right)\right)\right\rangle_{\partial T} \\
& -\frac{1}{2} \sum_{T \in \mathcal{T}_{h}} \sum_{i, j=1}^{d}\left\langle\left(\partial_{j} \sigma_{0}-\sigma_{g j}\right) n_{i}, a_{i j} u-\mathcal{Q}_{h}^{(s)}\left(a_{i j} \mathcal{Q}_{h}^{(s)} u\right)\right\rangle_{\partial T} \\
& +\sum_{T \in \mathcal{T}_{h}}\left(\mathcal{L}\left(\sigma_{0}\right), \mathcal{Q}_{h}^{(s)} u-u\right)_{T} .
\end{aligned}
$$

Proof. First of all, from 5.5 and 3.3 we have

$$
b\left(\epsilon_{h}, v\right)=b\left(\rho_{h}, v\right)=0, \quad \forall v \in W_{h, s},
$$

which gives 5.7 .

Next, notice that $\rho=0$. Thus, by using $(3.2)$ we arrive at

$$
\begin{aligned}
& s\left(\rho_{h}-Q_{h} \rho, \sigma\right)+b\left(\sigma, u_{h}-\mathcal{Q}_{h}^{(s)} u\right) \\
= & s\left(\rho_{h}, \sigma\right)+b\left(\sigma, u_{h}\right)-b\left(\sigma, \mathcal{Q}_{h}^{(s)} u\right) \\
= & -\left(f, \sigma_{0}\right)-b\left(\sigma, \mathcal{Q}_{h}^{(s)} u\right) .
\end{aligned}
$$

The rest of the proof shall deal with the term $b\left(\sigma, \mathcal{Q}_{h}^{(s)} u\right)$. To this end, we use Lemma 5.1 to obtain

$$
\begin{aligned}
b\left(\sigma, \mathcal{Q}_{h}^{(s)} u\right) & =\sum_{T \in \mathcal{T}_{h}}\left(\mathcal{L}_{w} \sigma, \mathcal{Q}_{h}^{(s)} u\right)_{T} \\
& =\sum_{T \in \mathcal{T}_{h}}\left(\mathcal{L} \sigma_{0}, \mathcal{Q}_{h}^{(s)} u\right)_{T}+R_{T}\left(\sigma, \mathcal{Q}_{h}^{(s)} u\right) \\
& =\sum_{T \in \mathcal{T}_{h}}\left(\mathcal{L} \sigma_{0}, u\right)_{T}+\left(\mathcal{L} \sigma_{0}, \mathcal{Q}_{h}^{(s)} u-u\right)_{T}+R_{T}\left(\sigma, \mathcal{Q}_{h}^{(s)} u\right)
\end{aligned}
$$


From the integration by parts we have

$$
\begin{aligned}
\sum_{T \in \mathcal{T}_{h}}\left(\mathcal{L} \sigma_{0}, u\right)_{T}= & \sum_{T \in \mathcal{T}_{h}}\left(\left(\boldsymbol{\mu} \cdot \nabla \sigma_{0}, u\right)_{T}+\frac{1}{2} \sum_{i, j=1}^{d}\left(a_{i j} \partial_{j i}^{2} \sigma_{0}, u\right)_{T}\right) \\
= & \sum_{T \in \mathcal{T}_{h}}\left(\sigma_{0},-\nabla \cdot(\boldsymbol{\mu} u)+\frac{1}{2} \sum_{i, j=1}^{d} \partial_{i j}^{2}\left(a_{i j} u\right)\right)_{T} \\
& +\sum_{T \in \mathcal{T}_{h}}\left\langle\sigma_{0}, \boldsymbol{\mu} u \cdot \mathbf{n}-\frac{1}{2} \sum_{i, j=1}^{d} n_{j} \partial_{i}\left(a_{i j} u\right)\right\rangle_{\partial T} \\
& +\sum_{T \in \mathcal{T}_{h}} \sum_{j=1}^{d}\left\langle\partial_{j} \sigma_{0}, \frac{1}{2} \sum_{i=1}^{d} a_{i j} n_{i} u\right\rangle_{\partial T} .
\end{aligned}
$$

As $u$ is the exact solution of (1.4) and $\sigma_{b}=0$ on $\partial \Omega$, we then have

$$
\begin{aligned}
\sum_{T \in \mathcal{T}_{h}}\left\langle\sigma_{b}, \boldsymbol{\mu} u \cdot \mathbf{n}-\frac{1}{2} \sum_{i, j=1}^{d} n_{j} \partial_{i}\left(a_{i j} u\right)\right\rangle_{\partial T} & =0 \\
\sum_{T \in \mathcal{T}_{h}} \sum_{j=1}^{d}\left\langle\sigma_{g j}, \frac{1}{2} \sum_{i=1}^{d} a_{i j} n_{i} u\right\rangle_{\partial T} & =0 .
\end{aligned}
$$

By combining 5.11) with 5.12 and 5.13 we arrive at

$$
\begin{aligned}
\sum_{T \in \mathcal{T}_{h}}\left(\mathcal{L} \sigma_{0}, u\right)_{T}= & -\left(\sigma_{0}, f\right)+\sum_{T \in \mathcal{T}_{h}}\left\langle\sigma_{0}-\sigma_{b}, \boldsymbol{\mu} u \cdot \mathbf{n}-\frac{1}{2} \sum_{i, j=1}^{d} n_{j} \partial_{i}\left(a_{i j} u\right)\right\rangle_{\partial T} \\
& +\sum_{T \in \mathcal{T}_{h}} \sum_{j=1}^{d}\left\langle\partial_{j} \sigma_{0}-\sigma_{g j}, \frac{1}{2} \sum_{i=1}^{d} a_{i j} n_{i} u\right\rangle_{\partial T} .
\end{aligned}
$$

Finally, substituting (5.14) and $(5.10)$ into $(5.9)$ gives rise to the error equation $(5.6)$ after regrouping the reminding terms. This completes the proof of the lemma. $\square$

6. Error Estimates. The goal of this section is to establish some error estimates for the numerical solutions arising from the primal-dual weak Galerkin finite element scheme 3.2 - -3.3 . The key to our error analysis is the error equations (5.6)-(5.7) and the inf-sup condition derived in Lemma 4.2.

6.1. Main results. We first show that the semi-norm induced from the stabilizer $s(\cdot, \cdot)$ is indeed a norm in the subspace $V_{h, k}^{0}$ consisting of weak functions with vanishing boundary value.

Lemma 6.1. The semi-norm $\|\cdot\|_{w}$ given in (4.4) defines a norm in the linear space $V_{h, k}^{0}$.

Proof. It suffices to verify the positivity property for $\|\cdot\|_{w}$. Let $\rho \in V_{h, k}^{0}$ be such that $\|\rho\|_{w}=0$. It follows that $s(\rho, \rho)=0$, and hence $\rho_{0}=\rho_{b}$ and $\nabla \rho_{0}=\boldsymbol{\rho}_{g}$ on each $\partial T$. Furthermore, we have $\mathcal{L}\left(\rho_{0}\right)=0$ on each element $T \in \mathcal{T}_{h}$. Thus, $\rho_{0} \in C_{0}^{1}(\Omega)$ and satisfies

$$
\mathcal{L} \rho_{0}=0, \quad \text { in } \Omega
$$


It follows that $\rho_{0} \equiv 0$, and hence $\rho \equiv 0$. This completes the proof. $\square$

Our main error estimate can be stated as follows.

THEOREM 6.2. Let $u$ and $\left(u_{h} ; \rho_{h}\right) \in W_{h, s} \times V_{h, k}^{0}$ be the solutions of (1.4) and (3.2)-(3.3), respectively. Let $m \in[2, k+1]$ if $s=k-1$ and $m \in[2, k]$ if $s=$ $k-2$. Assume that the coefficient tensor $a(x)$ and the drift vector $\boldsymbol{\mu}$ are uniformly piecewise smooth up to order $m-1$ in $\Omega$ with respect to the finite element partition $\mathcal{T}_{h}$. Additionally, assume that the exact solution $u$ is sufficiently regular such that $u \in H^{m-1}(\Omega) \cap H^{2}(\Omega)$. Then, we have

$$
\left\|\rho_{h}\right\|_{w}+\left\|u_{h}-\mathcal{Q}_{h}^{(s)} u\right\| \lesssim h^{m-1}\left(\|u\|_{m-1}+h \delta_{m, 2}\|u\|_{2}\right),
$$

provided that the meshsize $h<h_{0}$ holds true for a sufficiently small, but fixed $h_{0}>0$. Moreover, one has the following optimal order error estimate in $L^{2}$ :

$$
\left\|u-u_{h}\right\| \lesssim h^{m-1}\left(\|u\|_{m-1}+h \delta_{m, 2}\|u\|_{2}\right) .
$$

Proof. By letting $\sigma=\epsilon_{h}$ in the error equation (5.6) and using (5.7) we arrive at

$$
s\left(\epsilon_{h}, \epsilon_{h}\right)=\ell_{u}\left(\epsilon_{h}\right) .
$$

To deal with the term on the right-hand side of (6.3), we use the Cauchy-Schwarz inequality, the representation (5.8), and the estimates (7.5)-(7.8) to obtain

$$
\begin{aligned}
& \left|\ell_{u}(\sigma)\right| \\
\leq & \left(\sum_{T \in \mathcal{T}_{h}} h_{T}^{-3}\left\|\sigma_{0}-\sigma_{b}\right\|_{\partial T}^{2}\right)^{\frac{1}{2}}\left(\sum_{T \in \mathcal{T}_{h}} h_{T}^{3}\left\|\left(\boldsymbol{\mu} u-\mathcal{Q}_{h}^{(k-1)}\left(\boldsymbol{\mu} \mathcal{Q}_{h}^{(s)} u\right)\right) \cdot \mathbf{n}\right\|_{\partial T}^{2}\right)^{\frac{1}{2}} \\
+ & \left(\sum_{T \in \mathcal{T}_{h}} \sum_{j=1}^{d} h_{T}^{-3}\left\|\sigma_{0}-\sigma_{b}\right\|_{\partial T}^{2}\right)^{\frac{1}{2}}\left(\sum_{T \in \mathcal{T}_{h}} \sum_{i, j=1}^{d} h_{T}^{3}\left\|\partial_{i}\left(a_{i j} u-\mathcal{Q}_{h}^{(s)}\left(a_{i j} \mathcal{Q}_{h}^{(s)} u\right)\right)\right\|_{\partial T}^{2}\right)^{\frac{1}{2}} \\
+ & \left(\sum_{T \in \mathcal{T}_{h}} \sum_{j=1}^{d} h_{T}^{-1}\left\|\partial_{j} \sigma_{0}-\sigma_{g j}\right\|_{\partial T}^{2}\right)^{\frac{1}{2}}\left(\sum_{T \in \mathcal{T}_{h}} \sum_{i, j=1}^{d} h_{T}\left\|a_{i j} u-\mathcal{Q}_{h}^{(s)}\left(a_{i j} \mathcal{Q}_{h}^{(s)} u\right)\right\|_{\partial T}^{2}\right)^{\frac{1}{2}} \\
+ & \left(\sum_{T \in \mathcal{T}_{h}}\left\|\mathcal{L}\left(\sigma_{0}\right)\right\|_{T}^{2}\right)^{\frac{1}{2}}\left(\sum_{T \in \mathcal{T}_{h}}\left\|\mathcal{Q}_{h}^{(s)} u-u\right\|_{T}^{2}\right)^{\frac{1}{2}} \\
\lesssim & h^{k}\|u\|_{k-1}\|\sigma\|_{w}+h^{k-1}\left(\|u\|_{k-1}+h \delta_{k, 2}\|u\|_{2}\right)\|\sigma\|_{w} \\
& +h^{k-1}\|u\|_{k-1}\|\sigma\|_{w}+h^{k-1}\|u\|_{k-1}\|\sigma\|_{w} \\
\lesssim & h^{k-1}\left(\|u\|_{k-1}+h \delta_{k, 2}\|u\|_{2}\right)\|\sigma\|_{w},
\end{aligned}
$$

for any $\sigma \in V_{h, k}^{0}$. Now substituting the above estimate into 6.3 yields the following error estimate:

$$
\left\|\epsilon_{h}\right\|_{w}^{2} \lesssim h^{k-1}\left(\|u\|_{k-1}+h \delta_{k, 2}\|u\|_{2}\right)\left\|\epsilon_{h}\right\|_{w}
$$

which leads to

$$
\left\|\epsilon_{h}\right\|_{w} \lesssim h^{k-1}\left(\|u\|_{k-1}+h \delta_{k, 2}\|u\|_{2}\right)
$$


Next, for the error function $e_{h}=u_{h}-\mathcal{Q}_{h}^{(k-2)} u$, from the inf-sup condition (4.5)4.6 there exists a $\sigma \in V_{h, k}^{0}$ such that

$$
\frac{1}{2}\left\|e_{h}\right\|^{2} \leq\left|b\left(e_{h}, \sigma\right)\right|, \quad\|\sigma\|_{w} \leq \beta\left\|e_{h}\right\| .
$$

On the other hand, the error equation (5.6) implies

$$
b\left(e_{h}, \sigma\right)=\ell_{u}(\sigma)-s\left(\epsilon_{h}, \sigma\right) .
$$

It follows that

$$
\begin{aligned}
\left|b\left(e_{h}, \sigma\right)\right| & \leq\left|\ell_{u}(\sigma)\right|+\left\|\epsilon_{h}\right\|_{w}\|\sigma\|_{w} \\
& \lesssim h^{k-1}\left(\|u\|_{k-1}+h \delta_{k, 2}\|u\|_{2}\right)\|\sigma\|_{w} .
\end{aligned}
$$

Combing (6.5) with 6.6 gives rise to the following error estimate

$$
\frac{1}{2}\left\|e_{h}\right\|^{2} \lesssim h^{k-1}\left(\|u\|_{k-1}+h \delta_{k, 2}\|u\|_{2}\right)\left\|e_{h}\right\|
$$

which is

$$
\left\|e_{h}\right\| \lesssim h^{k-1}\left(\|u\|_{k-1}+h \delta_{k, 2}\|u\|_{2}\right) .
$$

The desired error estimate (6.1) is a direct result of (6.4) and 6.7). This completes the proof of the theorem.

In Table 6.1, we provide a summary for the rate of convergence for the numerical approximation $u_{h}$ arising from the WG scheme (3.2)- $(3.3)$. The first line of the table indicates the type of elements used in the numerical scheme. Recall that the space $W_{h, s}$ was employed for approximating $u_{h}$, while $V_{h, k}$ was for the auxiliary variable $\rho_{h}$. Although the solution $u_{h}$ is the quantity of major interest in this application, we believe that the auxiliary variable $\rho_{h}$ might provide some useful information for the design of error estimators for $u_{h}$. The second row of the table shows an optimal order of convergence for $u_{h}$ in the usual $L^{2}$-norm; i.e., a convergence of order $k$ when piecewise polynomials of degree $k-1$ are used.

TABLE 6.1

Convergence for the primal-dual weak Galerkin finite element method

\begin{tabular}{|c|c|c|}
\hline & $V_{h, k} \times W_{h, k-2}, k \geq 2$ & $V_{h, k} \times W_{h, k-1}, k \geq 1$ \\
\hline$\left\|u-u_{h}\right\|$ & $h^{k-1}$ & $h^{k}$ \\
\hline
\end{tabular}

6.2. Extension to $C^{0}$-type elements. By $C^{0}$-type elements, we mean a special class of finite element spaces $V_{h, k}$ consisting of weak finite element functions $v=\left\{v_{0}, v_{b}, \mathbf{v}_{g}\right\}$ where $v_{b}=\left.v_{0}\right|_{\partial T}$ on each element $T \in \mathcal{T}_{h}$. Analogously, $C^{-1}$-type elements refer to the general case of $v=\left\{v_{0}, v_{b}, \mathbf{v}_{g}\right\} \in V_{h, k}$ for which $v_{b}$ is totally independent of $v_{0}$. It is clear that $C^{0}$-type finite element schemes involve less number of degrees of freedom than $C^{-1}$-type, as the boundary component $v_{b}$ can be obviously eliminated from the list of unknowns. However, $C^{0}$-type elements would impose more limitations on the geometry of the finite element partition $\mathcal{T}_{h}$.

The error estimates shown as in Theorem 6.2 can be extended to $C^{0}$-type triangular elements for $V_{h, k}$. The rest of this section shall explain some modifications 
necessary for such an extension. First of all, for $C^{0}$-type elements, the discrete weak second order partial derivative $\partial_{i j, w}^{2} v$ can be computed as a polynomial in $P_{s}(T)$ on each element $T$ by the following equation

$$
\left(\partial_{i j, w}^{2} v, \varphi\right)_{T}=-\left(\partial_{i} v_{0}, \partial_{j} \varphi\right)_{T}+\left\langle v_{g i}, \varphi n_{j}\right\rangle_{\partial T}, \quad \forall \varphi \in P_{s}(T) .
$$

For the convergence analysis to work, we need to have the error equations (5.6)-(5.7) which in turn require the commutative property 4.2 - 4.3 for a properly defined projection operator $Q_{h}$ given as in 4.1. As there is no change on the variable $\mathbf{v}_{g}$ and the space it lives in, the operator $\mathbf{Q}_{g}$ should remain unchanged as the usual $L^{2}$ projection into the space of polynomials of degree $k-1$ on each piece of $\partial T$. However, the operator $Q_{0}$ must be modified by using the interpolation operator $\tilde{I}_{k}$ given as in Lemma A.3 of [14]. For 2D triangular elements, this interplant polynomial $\tilde{I}_{k} v \in P_{k}(T)$ satisfies

$$
\begin{array}{ll}
\int_{e}\left(v-\tilde{I}_{k} v\right) \phi d s=0 & \forall \phi \in P_{k-2}(e), \quad \forall \text { side } e \text { of } T \\
\int_{T}\left(v-\tilde{I}_{k} v\right) \phi d s=0 & \forall \phi \in P_{k-3}(T) .
\end{array}
$$

From the integration by parts, 6.9, and 6.10, we then obtain

$$
\begin{aligned}
\left(\partial_{i} \tilde{I}_{k} v, \partial_{j} \varphi\right)_{T} & =-\left(\tilde{I}_{k} v, \partial_{j i}^{2} \varphi\right)_{T}+\left\langle\tilde{I}_{k} v, \partial_{j} \varphi n_{i}\right\rangle_{\partial T} \\
& =-\left(v, \partial_{j i}^{2} \varphi\right)_{T}+\left\langle v, \partial_{j} \varphi n_{i}\right\rangle_{\partial T} \\
& =\left(\partial_{i} v, \partial_{j} \varphi\right)_{T}
\end{aligned}
$$

for all $\varphi \in P_{k-1}(T)$. Thus, from 6.8, 6.11, and the fact that $s \leq k-1$ we arrive at

$$
\begin{aligned}
\left(\partial_{i j, w}^{2} Q_{h} v, \varphi\right)_{T} & =-\left(\partial_{i} \tilde{I}_{k} v, \partial_{j} \varphi\right)_{T}+\left\langle\left(\mathbf{Q}_{g} \nabla v\right)_{i}, \varphi n_{j}\right\rangle_{\partial T} \\
& =-\left(\partial_{i} v, \partial_{j} \varphi\right)_{T}+\left\langle(\nabla v)_{i}, \varphi n_{j}\right\rangle_{\partial T} \\
& =\left(\partial_{i j}^{2} v, \varphi\right)_{T} \\
& =\left(\mathcal{Q}_{h} \partial_{i j}^{2} v, \varphi\right)_{T}
\end{aligned}
$$

for all $\varphi \in P_{s}(T)$, which implies the commutative property 4.2 . Note that 4.3 ) clearly holds true as the weak gradient is identical with the strong gradient for $C^{0}$ type elements. Readers are encouraged to check out [20] for a detailed discussion on the use of $C^{0}$-type elements in the context of weak Galerkin finite element methods.

7. Error Estimates for $L^{2}$ Projections. Recall that $\mathcal{T}_{h}$ is a shape-regular finite element partition of the domain $\Omega$. For any $T \in \mathcal{T}_{h}$ and $\phi \in H^{1}(T)$, the following trace inequality holds true [32]

$$
\|\phi\|_{\partial T}^{2} \lesssim h_{T}^{-1}\|\phi\|_{T}^{2}+h_{T}\|\nabla \phi\|_{T}^{2} .
$$

If $\phi$ is a polynomial on the element $T \in \mathcal{T}_{h}$, then from the inverse inequality, we have 32,

$$
\|\phi\|_{\partial T}^{2} \lesssim h_{T}^{-1}\|\phi\|_{T}^{2}
$$


Lemma 7.1. [32] Let $\mathcal{T}_{h}$ be a finite element partition of $\Omega$ satisfying the shape regular assumption given in [32]. For $0 \leq t \leq \min (2, k)$, the following estimates hold true:

$$
\begin{array}{ll}
\sum_{T \in \mathcal{T}_{h}} h_{T}^{2 t}\left\|u-Q_{0} u\right\|_{t, T}^{2} \lesssim h^{2(m+1)}\|u\|_{m+1}^{2}, & m \in[t-1, k], k \geq 1, \\
\sum_{T \in \mathcal{T}_{h}} h_{T}^{2 t}\left\|u-\mathcal{Q}_{h}^{(k-1)} u\right\|_{t, T}^{2} \lesssim h^{2 m}\|u\|_{m}^{2}, & m \in[t, k], k \geq 1, \\
\sum_{T \in \mathcal{T}_{h}} h_{T}^{2 t}\left\|u-\mathcal{Q}_{h}^{(k-2)} u\right\|_{t, T}^{2} \lesssim h^{2 m}\|u\|_{m}^{2}, & m \in[t, k-1], k \geq 2 .
\end{array}
$$

Note that 7.5) is merely a different form of 7.4.

Lemma 7.2. Assume that the coefficient tensor a $(x)$ and the drift vector $\boldsymbol{\mu}$ are uniformly piecewise smooth up to order $m-1$ in $\Omega$ with respect to the finite element partition $\mathcal{T}_{h}$. Then for any $v \in H^{m-1}(\Omega) \cap H^{2}(\Omega)$, the following estimates hold true:

$$
\begin{gathered}
\left(\sum_{T \in \mathcal{T}_{h}} h_{T}^{3}\left\|\left(\boldsymbol{\mu} v-\mathcal{Q}_{h}^{(k-1)}\left(\boldsymbol{\mu} \mathcal{Q}_{h}^{(s)} v\right)\right) \cdot \mathbf{n}\right\|_{\partial T}^{2}\right)^{\frac{1}{2}} \lesssim h^{m}\|v\|_{m-1} \\
\left(\sum_{T \in \mathcal{T}_{h}} \sum_{i, j=1}^{d} h_{T}^{3}\left\|\partial_{i}\left(a_{i j} v-\mathcal{Q}_{h}^{(s)}\left(a_{i j} \mathcal{Q}_{h}^{(s)} v\right)\right)\right\|_{\partial T}^{2}\right)^{\frac{1}{2}} \\
\lesssim h^{m-1}\left(\|v\|_{m-1}+h \delta_{m, 2}\|v\|_{2}\right) ; \\
\left(\sum_{T \in \mathcal{T}_{h}} \sum_{i, j=1}^{d} h_{T}\left\|a_{i j} u-\mathcal{Q}_{h}^{(s)}\left(a_{i j} \mathcal{Q}_{h}^{(s)} u\right)\right\|_{\partial T}^{2}\right)^{\frac{1}{2}} \lesssim h^{m-1}\|u\|_{m-1}
\end{gathered}
$$

Here, $m$ is an integer satisfying $m \in[2, k+1]$ if $s=k-1$ and $m \in[2, k]$ if $s=k-2$, and $\delta_{m, 2}$ is the usual Kronecker's delta with value 1 when $m=2$ and value 0 otherwise. have

Proof. To prove (7.6), from the trace inequality $(7.1)$ and the estimate 7.5 we

$$
\begin{aligned}
& \sum_{T \in \mathcal{T}_{h}} h_{T}^{3}\left\|\left(\boldsymbol{\mu} v-\mathcal{Q}_{h}^{(k-1)}\left(\boldsymbol{\mu} \mathcal{Q}_{h}^{(s)} v\right)\right) \cdot \mathbf{n}\right\|_{\partial T}^{2} \\
\lesssim & \sum_{T \in \mathcal{T}_{h}} h_{T}^{2}\left\|\boldsymbol{\mu} v-\mathcal{Q}_{h}^{(k-1)}\left(\boldsymbol{\mu} \mathcal{Q}_{h}^{(s)} v\right)\right\|_{T}^{2}+h_{T}^{4}\left\|\boldsymbol{\mu} v-\mathcal{Q}_{h}^{(k-1)}\left(\boldsymbol{\mu} \mathcal{Q}_{h}^{(s)} v\right)\right\|_{1, T}^{2} \\
\lesssim & \sum_{T \in \mathcal{T}_{h}} h_{T}^{2}\left\|\boldsymbol{\mu} v-\mathcal{Q}_{h}^{(k-1)}(\boldsymbol{\mu} v)\right\|_{T}^{2}+h_{T}^{2}\left\|\mathcal{Q}_{h}^{(k-1)}(\boldsymbol{\mu} v)-\mathcal{Q}_{h}^{(k-1)}\left(\boldsymbol{\mu} \mathcal{Q}_{h}^{(s)} v\right)\right\|_{T}^{2} \\
& \quad+h_{T}^{4}\left\|\boldsymbol{\mu} v-\mathcal{Q}_{h}^{(k-1)}(\boldsymbol{\mu} v)\right\|_{1, T}^{2}+h_{T}^{4}\left\|\mathcal{Q}_{h}^{(k-1)}(\boldsymbol{\mu} v)-\mathcal{Q}_{h}^{(k-1)}\left(\boldsymbol{\mu} \mathcal{Q}_{h}^{(s)} v\right)\right\|_{1, T}^{2} \\
\lesssim & \sum_{T \in \mathcal{T}_{h}} h_{T}^{2}\left\|\boldsymbol{\mu} v-\mathcal{Q}_{h}^{(k-1)}(\boldsymbol{\mu} v)\right\|_{T}^{2}+h_{T}^{2}\left\|\boldsymbol{\mu} v-\boldsymbol{\mu} \mathcal{Q}_{h}^{(s)} v\right\|_{T}^{2} \\
& \quad+h_{T}^{4}\left\|\boldsymbol{\mu} v-\mathcal{Q}_{h}^{(k-1)}(\boldsymbol{\mu} v)\right\|_{1, T}^{2}+h_{T}^{4}\left\|\boldsymbol{\mu} v-\boldsymbol{\mu} \mathcal{Q}_{h}^{(s)} v\right\|_{1, T}^{2} \\
\lesssim & h^{2 m}\|v\|_{m-1}^{2}
\end{aligned}
$$

for $m \in[2, k+1]$ when $s=k-1$ and $m \in[2, k]$ when $s=k-2$. 
As to (7.7), we use the trace inequality (7.1) and the estimate 7.5 to obtain

$$
\begin{aligned}
& \sum_{T \in \mathcal{T}_{h}} \sum_{i, j=1}^{d} h_{T}^{3}\left\|\partial_{i}\left(a_{i j} v-\mathcal{Q}_{h}^{(s)}\left(a_{i j} \mathcal{Q}_{h}^{(s)} v\right)\right)\right\|_{\partial T}^{2} \\
\lesssim & \sum_{T \in \mathcal{T}_{h}} \sum_{i, j=1}^{d} h_{T}^{2}\left\|\partial_{i}\left(a_{i j} v-\mathcal{Q}_{h}^{(s)}\left(a_{i j} \mathcal{Q}_{h}^{(s)} v\right)\right)\right\|_{T}^{2}+h_{T}^{4}\left\|\partial_{i}\left(a_{i j} v-\mathcal{Q}_{h}^{(s)}\left(a_{i j} \mathcal{Q}_{h}^{(s)} v\right)\right)\right\|_{1, T}^{2} \\
\lesssim & \sum_{T \in \mathcal{T}_{h}} \sum_{i, j=1}^{d} h_{T}^{2}\left\|\partial_{i}\left(a_{i j} v-\mathcal{Q}_{h}^{(s)}\left(a_{i j} v\right)\right)\right\|_{T}^{2}+h_{T}^{2}\left\|\partial_{i}\left(\mathcal{Q}_{h}^{(s)}\left(a_{i j} v\right)-\mathcal{Q}_{h}^{(s)}\left(a_{i j} \mathcal{Q}_{h}^{(s)} v\right)\right)\right\|_{T}^{2} \\
& \quad+h_{T}^{4}\left\|\partial_{i}\left(a_{i j} v-\mathcal{Q}_{h}^{(s)}\left(a_{i j} v\right)\right)\right\|_{1, T}^{2}+h_{T}^{4}\left\|\partial_{i}\left(\mathcal{Q}_{h}^{(s)}\left(a_{i j} v\right)-\mathcal{Q}_{h}^{(s)}\left(a_{i j} \mathcal{Q}_{h}^{(s)} v\right)\right)\right\|_{1, T}^{2} \\
\lesssim & h^{2 m-2}\left(\|v\|_{m-1}^{2}+h^{2} \delta_{m, 2}\|v\|_{2}^{2}\right),
\end{aligned}
$$

for $m \in[2, k+1]$ when $s=k-1$ and $m \in[2, k]$ when $s=k-2$.

Finally for (7.8), we again use the trace inequality $(7.1)$ and the estimate 7.5$)$ to obtain

$$
\begin{aligned}
& \sum_{T \in \mathcal{T}_{h}} \sum_{i, j=1}^{d} h_{T}\left\|a_{i j} v-\mathcal{Q}_{h}^{(s)}\left(a_{i j} \mathcal{Q}_{h}^{(s)} v\right)\right\|_{\partial T}^{2} \\
\lesssim & \sum_{T \in \mathcal{T}_{h}} \sum_{i, j=1}^{d}\left\|a_{i j} v-\mathcal{Q}_{h}^{(s)}\left(a_{i j} \mathcal{Q}_{h}^{(s)} v\right)\right\|_{T}^{2}+h_{T}^{2}\left\|a_{i j} v-\mathcal{Q}_{h}^{(s)}\left(a_{i j} \mathcal{Q}_{h}^{(s)} v\right)\right\|_{1, T}^{2} \\
\lesssim & \sum_{T \in \mathcal{T}_{h}} \sum_{i, j=1}^{d}\left\|a_{i j} v-\mathcal{Q}_{h}^{(s)}\left(a_{i j} v\right)\right\|_{T}^{2}+\left\|\mathcal{Q}_{h}^{(s)}\left(a_{i j} v\right)-\mathcal{Q}_{h}^{(s)}\left(a_{i j} \mathcal{Q}_{h}^{(s)} v\right)\right\|_{T}^{2} \\
& \quad+h_{T}^{2}\left\|a_{i j} v-\mathcal{Q}_{h}^{(s)}\left(a_{i j} v\right)\right\|_{1, T}^{2}+h_{T}^{2}\left\|\mathcal{Q}_{h}^{(s)}\left(a_{i j} v\right)-\mathcal{Q}_{h}^{(s)}\left(a_{i j} \mathcal{Q}_{h}^{(s)} v\right)\right\|_{1, T}^{2} \\
\lesssim & \sum_{T \in \mathcal{T}_{h}} \sum_{i, j=1}^{d}\left\|a_{i j} v-\mathcal{Q}_{h}^{(s)}\left(a_{i j} v\right)\right\|_{T}^{2}+\left\|a_{i j} v-a_{i j} \mathcal{Q}_{h}^{(s)} v\right\|_{T}^{2} \\
\quad & \quad+h_{T}^{2}\left\|a_{i j} v-\mathcal{Q}_{h}^{(s)}\left(a_{i j} v\right)\right\|_{1, T}^{2}+h_{T}^{2}\left\|a_{i j} v-a_{i j} \mathcal{Q}_{h}^{(s)} v\right\|_{1, T}^{2} \\
\lesssim & h^{2 m-2}\|v\|_{m-1}^{2},
\end{aligned}
$$

where $m \in[2, k+1]$ if $s=k-1$ and $m \in[2, k]$ if $s=k-2$. This completes the proof of the lemma.

8. Numerical Results. The goal of this section is to present some numerical results for the finite element scheme (3.2)-(3.3). Our test problem seeks $u$ satisfying

$$
\begin{aligned}
\nabla \cdot(\boldsymbol{\mu} u)-\frac{1}{2} \sum_{i, j=1}^{d} \partial_{i j}^{2}\left(a_{i j} u\right) & =f, \quad \text { in } \Omega, \\
u & =g, \quad \text { on } \partial \Omega,
\end{aligned}
$$

where $\Omega$ is a polygonal domain in $2 \mathrm{D}$. As the problem 8.1 has non-homogeneous Dirichlet data on the boundary, the weak formulation (1.4) must be modified accordingly so that the weak solution $u=u(x) \in L^{2}(\Omega)$ is given by the following equation

$$
\int_{\Omega} u \mathcal{L}(v) d x=\frac{1}{2} \sum_{i, j=1}^{d} \int_{\partial \Omega} g a_{i j} n_{i} \partial_{j} v d s-\int_{\Omega} f v d x, \quad \forall v \in H^{2}(\Omega) \cap H_{0}^{1}(\Omega) .
$$


The corresponding primal-dual weak Galerkin finite element scheme seeks $\left(u_{h} ; \rho_{h}\right) \in$ $W_{h, s} \times V_{h, k}^{0}$ satisfying

$$
\begin{array}{rlrl}
s\left(\rho_{h}, \sigma\right)+b\left(\sigma, u_{h}\right) & =\frac{1}{2} \sum_{i, j=1}^{d}\left\langle a_{i j} g, \sigma_{g j} n_{i}\right\rangle_{\partial \Omega}-\left(f, \sigma_{0}\right), & & \forall \sigma \in V_{h, k}^{0}, \\
b\left(\rho_{h}, v\right) & =0, & \forall v \in W_{h, s} .
\end{array}
$$

Our numerical implementation is based on the PD-WG scheme (8.3)-(8.4) with the element of order $k=2$ on uniformly triangular finite element partitions. This configuration corresponds to the following selection for the finite element spaces:

$$
V_{h, 2}=\left\{\rho=\left\{\rho_{0}, \rho_{b}, \boldsymbol{\rho}_{g}\right\}: \rho_{0} \in P_{2}(T), \rho_{b} \in P_{2}(e), \boldsymbol{\rho}_{g} \in\left[P_{1}(e)\right]^{2}, e \subset \partial T, T \in \mathcal{T}_{h}\right\},
$$

and

$$
W_{h, s}=\left\{w:\left.w\right|_{T} \in P_{s}(T), \forall T \in \mathcal{T}_{h}\right\}, \quad s=0 \text { or } 1 .
$$

For simplicity, our numerical experiments will be conducted for only $C^{0}$-type elements for which $\rho_{b}$ is identical with the trace of $\rho_{0}$ on $\partial T$ for any $T \in \mathcal{T}_{h}$. For convenience, the $C^{0}$-type WG element with $s=1$ (i.e., $W_{h, 1}$ ) shall be named as the $P_{2}(T) /\left[P_{1}(\partial T)\right]^{2} / P_{1}(T)$ element. Analogously, the case corresponding to $s=0$ (i.e., $\left.W_{h, 0}\right)$ is named as $P_{2}(T) /\left[P_{1}(\partial T)\right]^{2} / P_{0}(T)$.

Two polygonal domains are considered in the numerical experiments, with the first one being the unit square $\Omega=(0,1)^{2}$ and the second an L-shaped region with vertices $A_{0}=(0,0), A_{1}=(2,0), A_{2}=(2,1), A_{3}=(1,1), A_{4}=(1,2)$, and $A_{5}=(0,2)$. Note that the error estimates developed in the previous section are applicable to the square domain, but not to the L-shaped domain. Nevertheless, our primal-dual weak Galerkin finite element scheme is well formulated on any domains. The L-shaped domain is chosen just for the purposes of demonstrating the performance of the algorithm for cases for which theory has not been developed.

The finite element partitions in our computation are obtained through a simple uniform refinement procedure as follows. Given an initial coarse triangulation of the domain, a sequence of triangulations are obtained successively through a uniform refinement that divides each coarse level triangle into four congruent sub-triangles by connecting the three mid-points on the edges. We use $\rho_{h}=\left\{\rho_{0}, \boldsymbol{\rho}_{q}\right\} \in V_{h, 2}$ and $u_{h} \in W_{h, s}, s=0,1$, to denote the numerical solutions arising from $(3.2)-(3.3)$. The numerical solutions are compared with carefully chosen interpolations of the exact solution in various norms. In particular, the primal variable $u_{h}$ is compared with the exact solution $u$ on each element at either three vertices (for $s=1$ ) or the center (for $s=0$ ), which is known as the nodal point interpolation and is denoted as $I_{h} u$. The auxiliary variable $\rho_{h}$ is supposed to approximate the true solution $\rho=0$, and is therefore compared with $Q_{h} \rho=0$. The error functions are thus denoted as

$$
\epsilon_{h}=\rho_{h}-Q_{h} \rho \equiv\left\{\rho_{0}, \boldsymbol{\rho}_{g}\right\}, \quad e_{h}=u_{h}-I_{h} u
$$


The following norms are applied in the computation for the error:

$$
\begin{aligned}
L^{2} \text { - norm: }\left\|\rho_{h}\right\|_{0} & =\left(\sum_{T \in \mathcal{T}_{h}} \int_{T}\left|\rho_{0}\right|^{2} d T\right)^{\frac{1}{2}}, \\
\text { Semi } H^{1} \text {-norm: } \quad\left\|\boldsymbol{\rho}_{h}\right\|_{1} & =\left(\sum_{T \in \mathcal{T}_{h}} h_{T} \int_{\partial T}\left|\boldsymbol{\rho}_{g}\right|^{2} d s\right)^{\frac{1}{2}}, \\
L^{2} \text {-norm: } \quad\left\|e_{h}\right\|_{0} & =\left(\sum_{T \in \mathcal{T}_{h}} \int_{T}\left|e_{h}\right|^{2} d T\right)^{\frac{1}{2}} .
\end{aligned}
$$

Tables 8.18 .2 illustrate the performance of $C^{0}$-type $P_{2}(T) /\left[P_{1}(\partial T)\right]^{2} / P_{1}(T)$ element for the test problem (8.1) with exact solution given by $u=\sin \left(x_{1}\right) \sin \left(x_{2}\right)$ on the unit square domain and the L-shaped domain. The right-hand side function and the Dirichlet boundary condition are chosen to match the exact solution. Our numerical results indicate that the convergence rate for the solution $u_{h}$ is of order $r=2$ in the discrete $L^{2}$-norm on both the unit square domain and the L-shaped domain. The result is in great consistency with the theoretical rate of convergence for $u_{h}$.

TABLE 8.1

Numerical rates of convergence for the $C^{0}-P_{2}(T) /\left[P_{1}(\partial T)\right]^{2} / P_{1}(T)$ element applied to problem 8.1) with exact solution $u=\sin \left(x_{1}\right) \sin \left(x_{2}\right)$ on $\Omega=(0,1)^{2}$. The coefficient matrix is $a_{11}=3$, $a_{12}=a_{21}=1$, and $a_{22}=2$. The drift vector is $\mu=[1,1]$.

\begin{tabular}{|c|c|c|c|c|c|c|}
\hline $1 / h$ & $\left\|\rho_{h}\right\|_{0}$ & order & $\left\|\boldsymbol{\rho}_{g}\right\|_{1}$ & order & $\left\|u_{h}-I_{h} u\right\|_{0}$ & order \\
\hline 1 & $3.52 \mathrm{e}-01$ & & $4.02 \mathrm{e}-00$ & & $2.11 \mathrm{e}-01$ & \\
\hline 2 & $4.09 \mathrm{e}-02$ & 3.11 & $7.16 \mathrm{e}-01$ & 2.49 & $5.55 \mathrm{e}-02$ & 1.93 \\
\hline 4 & $3.77 \mathrm{e}-03$ & 3.44 & $1.22 \mathrm{e}-01$ & 2.55 & $1.42 \mathrm{e}-02$ & 1.97 \\
\hline 8 & $2.78 \mathrm{e}-04$ & 3.76 & $1.79 \mathrm{e}-02$ & 2.79 & $3.57 \mathrm{e}-03$ & 1.99 \\
\hline 16 & $1.87 \mathrm{e}-05$ & 3.90 & $2.36 \mathrm{e}-03$ & 2.91 & $8.93 \mathrm{e}-04$ & 2.00 \\
\hline 32 & $1.20 \mathrm{e}-06$ & 3.95 & $3.04 \mathrm{e}-04$ & 2.96 & $2.23 \mathrm{e}-04$ & 2.00 \\
\hline
\end{tabular}

TABLE 8.2

Numerical rates of convergence for the $C^{0}-P_{2}(T) /\left[P_{1}(\partial T)\right]^{2} / P_{1}(T)$ element applied to the problem 8.1) with exact solution $u=\sin \left(x_{1}\right) \sin \left(x_{2}\right)$ on the L-shaped domain. The coefficient matrix is $a_{11}=3, a_{12}=a_{21}=1$, and $a_{22}=2$. The drift vector is $\mu=[1,1]$.

\begin{tabular}{|c|c|c|c|c|c|c|}
\hline $1 / h$ & $\left\|\rho_{h}\right\|_{0}$ & order & $\left\|\boldsymbol{\rho}_{g}\right\|_{1}$ & order & $\left\|u_{h}-I_{h} u\right\|_{0}$ & order \\
\hline 1 & $5.33 \mathrm{e}-01$ & & $5.20 \mathrm{e}-00$ & & $3.07 \mathrm{e}-01$ & \\
\hline 2 & $7.77 \mathrm{e}-02$ & 2.78 & $1.18 \mathrm{e}-00$ & 2.15 & $8.03 \mathrm{e}-02$ & 1.94 \\
\hline 4 & $6.42 \mathrm{e}-03$ & 3.60 & $1.90 \mathrm{e}-01$ & 2.63 & $2.04 \mathrm{e}-02$ & 1.98 \\
\hline 8 & $4.49 \mathrm{e}-04$ & 3.84 & $2.65 \mathrm{e}-02$ & 2.84 & $5.11 \mathrm{e}-03$ & 2.00 \\
\hline 16 & $2.95 \mathrm{e}-05$ & 3.93 & $3.47 \mathrm{e}-03$ & 2.93 & $1.27 \mathrm{e}-03$ & 2.00 \\
\hline 32 & $1.89 \mathrm{e}-06$ & 3.97 & $4.44 \mathrm{e}-04$ & 2.97 & $3.18 \mathrm{e}-04$ & 2.00 \\
\hline
\end{tabular}

Tables 8.38 .4 illustrate the performance of the $C^{0}$-type $P_{2}(T) /\left[P_{1}(\partial T)\right]^{2} / P_{0}(T)$ element for the test problem 8.1 with exact solution given by $u=\sin \left(x_{1}\right) \sin \left(x_{2}\right)$ on the unit square domain and the L-shaped domain with constant coefficients. The numerical results are in good consistency with what the theory predicts. 
TABLE 8.3

Numerical rates of convergence for the $C^{0}-P_{2}(T) /\left[P_{1}(\partial T)\right]^{2} / P_{0}(T)$ element applied to the problem 8.1) with exact solution $u=\sin \left(x_{1}\right) \sin \left(x_{2}\right)$ on $\Omega=(0,1)^{2}$. The coefficient matrix is $a_{11}=3, a_{12}=a_{21}=1$, and $a_{22}=2$. The drift vector is $\mu=[1,1]$.

\begin{tabular}{|c|c|c|c|c|c|c|}
\hline $1 / h$ & $\left\|\rho_{h}\right\|_{0}$ & order & $\left\|\boldsymbol{\rho}_{g}\right\|_{1}$ & order & $\left\|u_{h}-I_{h} u\right\|_{0}$ & order \\
\hline 1 & $9.82 \mathrm{e}-03$ & & $2.57 \mathrm{e}-01$ & & $1.03 \mathrm{e}-01$ & \\
\hline 2 & $4.79 \mathrm{e}-03$ & 1.04 & $6.98 \mathrm{e}-02$ & 1.88 & $4.38 \mathrm{e}-02$ & 1.23 \\
\hline 4 & $1.53 \mathrm{e}-03$ & 1.65 & $1.81 \mathrm{e}-02$ & 1.95 & $1.72 \mathrm{e}-02$ & 1.35 \\
\hline 8 & $3.76 \mathrm{e}-04$ & 2.02 & $4.43 \mathrm{e}-03$ & 2.03 & $7.74 \mathrm{e}-03$ & 1.15 \\
\hline 16 & $9.27 \mathrm{e}-05$ & 2.02 & $1.09 \mathrm{e}-03$ & 2.02 & $3.77 \mathrm{e}-03$ & 1.04 \\
\hline 32 & $2.31 \mathrm{e}-05$ & 2.01 & $2.72 \mathrm{e}-04$ & 2.01 & $1.87 \mathrm{e}-03$ & 1.01 \\
\hline
\end{tabular}

TABLE 8.4

Convergence rates for the $C^{0}-P_{2}(T) /\left[P_{1}(\partial T)\right]^{2} / P_{0}(T)$ element applied to problem 8.1) with exact solution $u=\sin \left(x_{1}\right) \sin \left(x_{2}\right)$ on the L-shaped domain. The coefficient matrix is $a_{11}=3$, $a_{12}=a_{21}=1$, and $a_{22}=2$. The drift vector is $\mu=[1,1]$.

\begin{tabular}{|c|c|c|c|c|c|c|}
\hline $1 / h$ & $\left\|\rho_{h}\right\|_{0}$ & order & $\left\|\boldsymbol{\rho}_{g}\right\|_{1}$ & order & $\left\|u_{h}-I_{h} u\right\|_{0}$ & order \\
\hline 1 & 0.0199 & & $5.01 \mathrm{e}-01$ & & $1.72 \mathrm{e}-01$ & \\
\hline 2 & 0.0165 & 0.269 & $1.39 \mathrm{e}-01$ & 1.85 & $7.65 \mathrm{e}-02$ & 1.17 \\
\hline 4 & 0.00470 & 1.81 & $3.60 \mathrm{e}-02$ & 1.95 & $3.37 \mathrm{e}-02$ & 1.18 \\
\hline 8 & $1.18 \mathrm{E}-03$ & 2.00 & $8.96 \mathrm{e}-03$ & 2.01 & $1.61 \mathrm{e}-02$ & 1.07 \\
\hline 16 & $2.93 \mathrm{E}-04$ & 2.01 & $2.23 \mathrm{e}-03$ & 2.01 & $7.92 \mathrm{e}-03$ & 1.02 \\
\hline 32 & $7.32 \mathrm{E}-05$ & 2.00 & $5.56 \mathrm{e}-04$ & 2.00 & $3.94 \mathrm{e}-03$ & 1.01 \\
\hline
\end{tabular}

Tables 8.58 .6 illustrate the performance of the $C^{0}$-type $P_{2}(T) /\left[P_{1}(\partial T)\right]^{2} / P_{0}(T)$ element for the test problem 8.1) on the unit square domain and the L-shaped domain. The exact solution is given by $u=\sin \left(x_{1}\right) \sin \left(x_{2}\right)$ and the differential operator has variable coefficients. The numerical rate of convergence for the primal-dual WG finite element method is consistent with the theory.

TABLE 8.5

Numerical rates of convergence for the $C^{0}-P_{2}(T) /\left[P_{1}(\partial T)\right]^{2} / P_{0}(T)$ element applied to problem 8.1) with exact solution $u=\sin \left(x_{1}\right) \sin \left(x_{2}\right)$ on $\Omega=(0,1)^{2}$. The coefficient matrix is given by $a_{11}=1+x_{1}^{2}, a_{12}=a_{21}=0.25 x_{1} x_{2}$, and $a_{22}=1+x_{2}^{2}$. The drift vector is $\mu=[x, y]$.

\begin{tabular}{|c|c|c|c|c|c|c|}
\hline $1 / h$ & $\left\|\rho_{h}\right\|_{0}$ & order & $\left\|\boldsymbol{\rho}_{g}\right\|_{1}$ & order & $\left\|u_{h}-I_{h} u\right\|_{0}$ & order \\
\hline 1 & $1.18 \mathrm{e}-02$ & & $1.94 \mathrm{e}-01$ & & $4.88 \mathrm{e}-02$ & \\
\hline 2 & $1.09 \mathrm{e}-03$ & 3.44 & $4.70 \mathrm{e}-02$ & 2.05 & $2.49 \mathrm{e}-02$ & 0.97 \\
\hline 4 & $3.13 \mathrm{e}-04$ & 1.81 & $1.13 \mathrm{e}-02$ & 2.05 & $1.14 \mathrm{e}-02$ & 1.13 \\
\hline 8 & $8.76 \mathrm{e}-05$ & 1.83 & $2.77 \mathrm{e}-03$ & 2.03 & $5.52 \mathrm{e}-03$ & 1.04 \\
\hline 16 & $2.25 \mathrm{e}-05$ & 1.96 & $6.85 \mathrm{e}-04$ & 2.02 & $2.74 \mathrm{e}-03$ & 1.01 \\
\hline 32 & $5.67 \mathrm{e}-06$ & 2.00 & $1.70 \mathrm{e}-04$ & 2.01 & $1.37 \mathrm{e}-03$ & 1.00 \\
\hline
\end{tabular}


TABLE 8.6

Numerical rates of convergence rates for the $C^{0}-P_{2}(T) /\left[P_{1}(\partial T)\right]^{2} / P_{0}(T)$ element applied to the problem (8.1) with exact solution $u=\sin \left(x_{1}\right) \sin \left(x_{2}\right)$ on the L-shaped domain. The coefficient matrix is given by $a_{11}=1+x_{1}^{2}, a_{12}=a_{21}=0.25 x_{1} x_{2}$, and $a_{22}=1+x_{2}^{2}$. The drift vector is $\mu=[x, y]$.

\begin{tabular}{|c|c|c|c|c|c|c|}
\hline $1 / h$ & $\left\|\rho_{h}\right\|_{0}$ & order & $\left\|\boldsymbol{\rho}_{g}\right\|_{1}$ & order & $\left\|u_{h}-I_{h} u\right\|_{0}$ & order \\
\hline 1 & $2.12 \mathrm{e}-02$ & & $5.39 \mathrm{e}-01$ & & $2.03 \mathrm{e}-01$ & \\
\hline 2 & $1.25 \mathrm{e}-02$ & 0.763 & $1.33 \mathrm{e}-01$ & 2.02 & $9.15 \mathrm{e}-02$ & 1.15 \\
\hline 4 & $3.09 \mathrm{e}-03$ & 2.01 & $3.24 \mathrm{e}-02$ & 2.04 & $4.23 \mathrm{e}-02$ & 1.11 \\
\hline 8 & $7.60 \mathrm{e}-04$ & 2.02 & $7.93 \mathrm{e}-03$ & 2.03 & $2.06 \mathrm{e}-02$ & 1.04 \\
\hline 16 & $1.89 \mathrm{e}-04$ & 2.01 & $1.96 \mathrm{e}-03$ & 2.02 & $1.02 \mathrm{e}-02$ & 1.01 \\
\hline 32 & $4.71 \mathrm{e}-05$ & 2.00 & $4.87 \mathrm{e}-04$ & 2.01 & $5.10 \mathrm{e}-03$ & 1.00 \\
\hline
\end{tabular}

Tables 8.78 .9 illustrate the performance of the $C^{0}$-type $P_{2}(T) /\left[P_{1}(\partial T)\right]^{2} / P_{1}(T)$ element for the test problem (8.1) when the parameter $\delta$ varies in the stabilizer $s(\cdot, \cdot)$. The exact solution is given by $u=\sin \left(x_{1}\right) \sin \left(x_{2}\right)$, and the domain in this test case is the unit square with constant coefficients and drifting. The results indicate that the convergence rate for the solution $u_{h}$ of the weak Galerkin algorithm $(3.2)-(3.3)$ is of order $r=2$ in the discrete $L^{2}$-norm for $u_{h}$ for different values of $\delta$. Table 8.10 illustrates the performance of the numerical scheme on the L-shaped domain with $\delta=10000$. It is interesting to note that the absolute error decreases as $\delta$ increases.

TABLE 8.7

Numerical rates of convergence for the $C^{0}-P_{2}(T) /\left[P_{1}(\partial T)\right]^{2} / P_{1}(T)$ element applied to the problem 8.1) with exact solution $u=\sin \left(x_{1}\right) \sin \left(x_{2}\right)$ on $\Omega=(0,1)^{2}$. The coefficient matrix is $a_{11}=3, a_{12}=a_{21}=1$, and $a_{22}=2$. The drift vector is $\mu=[1,1]$. The stabilizer parameter has value $\delta=0.1$.

\begin{tabular}{|c|c|c|c|c|c|c|}
\hline $1 / h$ & $\left\|\rho_{h}\right\|_{0}$ & order & $\left\|\boldsymbol{\rho}_{g}\right\|_{1}$ & order & $\left\|u_{h}-I_{h} u\right\|_{0}$ & order \\
\hline 1 & $2.22 \mathrm{E}-01$ & & $2.59 \mathrm{E}+00$ & & $1.40 \mathrm{E}-01$ & \\
\hline 2 & $2.12 \mathrm{E}-02$ & 3.39 & $4.51 \mathrm{E}-01$ & 2.52 & $4.58 \mathrm{E}-02$ & 1.61 \\
\hline 4 & $1.62 \mathrm{E}-03$ & 3.71 & $6.74 \mathrm{E}-02$ & 2.74 & $1.29 \mathrm{E}-02$ & 1.83 \\
\hline 8 & $1.09 \mathrm{E}-04$ & 3.89 & $8.97 \mathrm{E}-03$ & 2.91 & $3.39 \mathrm{E}-03$ & 1.93 \\
\hline 16 & $7.01 \mathrm{E}-06$ & 3.96 & $1.15 \mathrm{E}-03$ & 2.97 & $8.65 \mathrm{E}-04$ & 1.97 \\
\hline 32 & $4.41 \mathrm{E}-07$ & 3.99 & $1.44 \mathrm{E}-04$ & 2.99 & $2.18 \mathrm{E}-04$ & 1.99 \\
\hline
\end{tabular}

TABLE 8.8

Numerical rates of convergence for the $C^{0}-P_{2}(T) /\left[P_{1}(\partial T)\right]^{2} / P_{1}(T)$ element applied to the problem (8.1) with exact solution $u=\sin \left(x_{1}\right) \sin \left(x_{2}\right)$ on $\Omega=(0,1)^{2}$. The coefficient matrix is $a_{11}=3, a_{12}=a_{21}=1$, and $a_{22}=2$. The drift vector is $\mu=[1,1]$. The stabilizer parameter has value $\delta=1.0$.

\begin{tabular}{|c|c|c|c|c|c|c|}
\hline $1 / h$ & $\left\|\rho_{h}\right\|_{0}$ & order & $\left\|\boldsymbol{\rho}_{g}\right\|_{1}$ & order & $\left\|u_{h}-I_{h} u\right\|_{0}$ & order \\
\hline 1 & $5.11 \mathrm{e}-02$ & & $8.90 \mathrm{e}-01$ & & $5.58 \mathrm{e}-02$ & \\
\hline 2 & $8.51 \mathrm{e}-03$ & 2.59 & $1.83 \mathrm{e}-01$ & 2.28 & $2.20 \mathrm{e}-02$ & 1.35 \\
\hline 4 & $1.03 \mathrm{e}-03$ & 3.04 & $4.41 \mathrm{e}-02$ & 2.05 & $8.68 \mathrm{e}-03$ & 1.34 \\
\hline 8 & $9.02 \mathrm{e}-05$ & 3.52 & $7.65 \mathrm{e}-03$ & 2.53 & $2.88 \mathrm{e}-03$ & 1.59 \\
\hline 16 & $6.44 \mathrm{e}-06$ & 3.81 & $1.08 \mathrm{e}-03$ & 2.83 & $8.12 \mathrm{e}-04$ & 1.82 \\
\hline 32 & $4.24 \mathrm{e}-07$ & 3.93 & $1.40 \mathrm{e}-04$ & 2.94 & $2.13 \mathrm{e}-04$ & 1.93 \\
\hline
\end{tabular}


TABLE 8.9

Numerical rates of convergence for the $C^{0}-P_{2}(T) /\left[P_{1}(\partial T)\right]^{2} / P_{1}(T)$ element applied to the problem 8.1) with exact solution $u=\sin \left(x_{1}\right) \sin \left(x_{2}\right)$ on $\Omega=(0,1)^{2}$. The coefficient matrix is $a_{11}=3, a_{12}=a_{21}=1$, and $a_{22}=2$. The drift vector is $\mu=[1,1]$. The stabilizer parameter has value $\delta=10000$.

\begin{tabular}{|c|c|c|c|c|c|c|}
\hline $1 / h$ & $\left\|\rho_{h}\right\|_{0}$ & order & $\left\|\boldsymbol{\rho}_{g}\right\|_{1}$ & order & $\left\|u_{h}-I_{h} u\right\|_{0}$ & order \\
\hline 1 & $5.98 \mathrm{e}-06$ & & $6.78 \mathrm{e}-01$ & & $4.04 \mathrm{e}-02$ & \\
\hline 2 & $1.35 \mathrm{e}-06$ & 2.15 & $3.08 \mathrm{e}-02$ & 4.46 & $9.48 \mathrm{e}-03$ & 2.09 \\
\hline 4 & $6.40 \mathrm{e}-07$ & 1.08 & $1.39 \mathrm{e}-03$ & 4.47 & $2.05 \mathrm{e}-03$ & 2.21 \\
\hline 8 & $2.26 \mathrm{e}-07$ & 1.50 & $6.30 \mathrm{e}-05$ & 4.46 & $4.85 \mathrm{e}-04$ & 2.08 \\
\hline 16 & $5.97 \mathrm{e}-08$ & 1.92 & $6.38 \mathrm{e}-06$ & 3.30 & $1.21 \mathrm{e}-04$ & 2.01 \\
\hline 32 & $1.29 \mathrm{e}-08$ & 2.20 & $2.60 \mathrm{e}-06$ & 1.30 & $3.15 \mathrm{e}-05$ & 1.94 \\
\hline
\end{tabular}

TABLE 8.10

Numerical rates of convergence for the $C^{0}-P_{2}(T) /\left[P_{1}(\partial T)\right]^{2} / P_{1}(T)$ element applied to the problem 8.1) with exact solution $u=\sin \left(x_{1}\right) \sin \left(x_{2}\right)$ on the L-shaped domain. The coefficient matrix is $a_{11}=3, a_{12}=a_{21}=1$, and $a_{22}=2$. The drift vector is $\mu=[1,1]$. The stabilizer parameter has value $\delta=10000$.

\begin{tabular}{|c|c|c|c|c|c|c|}
\hline $1 / h$ & $\left\|\rho_{h}\right\|_{0}$ & order & $\left\|\boldsymbol{\rho}_{g}\right\|_{1}$ & order & $\left\|u_{h}-I_{h} u\right\|_{0}$ & order \\
\hline 1 & $8.31 \mathrm{e}-06$ & & $8.36 \mathrm{e}-01$ & & $1.33 \mathrm{e}-01$ & \\
\hline 2 & $6.90 \mathrm{e}-06$ & 0.269 & $3.64 \mathrm{e}-02$ & 4.52 & $2.51 \mathrm{e}-02$ & 2.40 \\
\hline 4 & $2.77 \mathrm{e}-06$ & 1.32 & $1.56 \mathrm{e}-03$ & 4.54 & $5.88 \mathrm{e}-03$ & 2.09 \\
\hline 8 & $7.97 \mathrm{e}-07$ & 1.79 & $8.03 \mathrm{e}-05$ & 4.28 & $1.45 \mathrm{e}-03$ & 2.03 \\
\hline 16 & $1.99 \mathrm{e}-07$ & 2.00 & $2.11 \mathrm{e}-05$ & 1.93 & $3.59 \mathrm{e}-04$ & 2.01 \\
\hline 32 & $4.35 \mathrm{e}-08$ & 2.20 & $9.34 \mathrm{e}-06$ & 1.18 & $8.99 \mathrm{e}-05$ & 2.00 \\
\hline
\end{tabular}

Figure 8.1 illustrates the performance of our numerical methods for a problem with discontinuous solution. This test problem has the following configuration: domain $\Omega=(-1,1) \times(-1,1)$, diffusion tensor $a=\alpha I$ with $\alpha=1$ for $x_{1}<0$ and $\alpha=2$ for $x_{1} \geq 0$, load function $f=0$, Dirichlet boundary condition $g=2$ for $x_{1}<0$ and $g=1$ for $x_{1}>0$. The numerical solutions are obtained by using piecewise linear functions for the primal variable; i.e., $s=1$ in the finite element space $W_{h, s}$. For this test problem, the exact solution is known to be $u=2$ for $x_{1}<0$ and $u=1$ for $x_{1}>0$ when the drift vector is zero. The plot indicates that the primal-dual weak Galerkin finite element solution is very consistent with the exact solution when $\boldsymbol{\mu}=0$ (see left plot). The right plot corresponds to the case of $\boldsymbol{\mu}=[1,1]$ for which no exact solution is known.

The primal-dual weak Galerkin finite element method $(3.2)-(3.3)$ was further applied to a test problem for which the exact solution is discontinuous along the $x_{2}$-axis. The configuration of this test problem is given as follows: domain $\Omega=$ $(-1,1) \times(-1,1)$, diffusion tensor $a=\alpha I$ with $\alpha=1$ for $x_{1}<0$ and $\alpha=2$ for $x_{1}>0$, load function $f=9 \sin \left(3 x_{2}\right)$, Dirichlet boudnary data $g=2 \sin \left(3 x_{2}\right)$ for $x_{1}<0$ and $g=\sin \left(3 x_{2}\right)$ for $x_{1}>0$, and drift vector $\boldsymbol{\mu}=0$. We use piecewise linear functions to approximate the primal variable, and the profile of the corresponding numerical solution is presented in Figure 8.2. The exact solution for this test problem is given by $u=2 \sin \left(3 x_{2}\right)$ for $x_{1}<0$ and $u=\sin \left(3 x_{2}\right)$ for $x_{1}>0$. The plot shows a great consistency between the numerical solution and the exact one. 

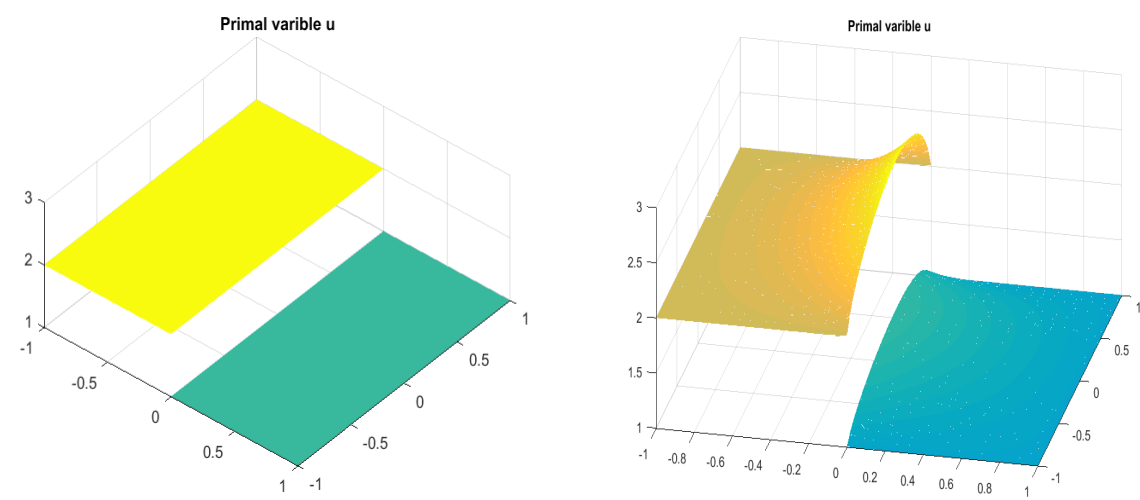

FIG. 8.1. Domain $\Omega=(-1,1) \times(-1,1)$, diffusion tensor $a=\alpha I$ with $\alpha=1$ for $x_{1}<0$ and $\alpha=2$ for $x_{1} \geq 0$, load function $f=0$, Dirichlet boundary data $g=2$ for $x_{1}<0$ and $g=1$ for $x_{1}>0$. Plot for primal variable $u_{h}$ : drift vector $\boldsymbol{\mu}=0$ (left), drift vector $\boldsymbol{\mu}=[1,1]$ (right).
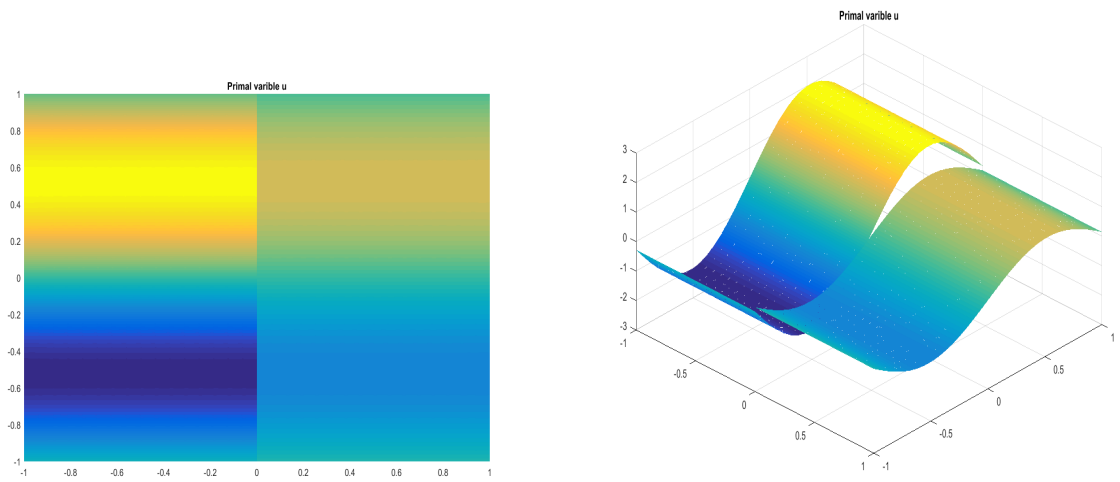

FIG. 8.2. Domain $\Omega=(-1,1) \times(-1,1)$, diffusion tensor $a=\alpha I$ with $\alpha=1$ for $x_{1}<0$ and $\alpha=2$ for $x_{1}>0$, load function $f=9 \sin \left(3 x_{2}\right)$, Dirichlet boudnary data $g=2 \sin \left(3 x_{2}\right)$ for $x_{1}<0$ and $g=\sin \left(3 x_{2}\right)$ for $x_{1}>0$, drift vector $\boldsymbol{\mu}=0$. Plot for primal variable $u_{h}$ : contour plot (left), surface plot (right).

Our last numerical experiment was conducted on a problem for which the exact solution is not only discontinuous, but also not known to us. This test problem has the following configuration: domain $\Omega=(-1,1) \times(-1,1)$, diffusion tensor $a=\alpha I$ with $\alpha=1$ in the first and third quadrant and $\alpha=10$ in the second and fourth quadrant, load function $f=\frac{1}{4}$, Dirichlet boundary data $g=0$, drift vector $\boldsymbol{\mu}=[1,1]$. The numerical solution for the primal variable can be seen in Figure 8.3. The left figure is the contour plot, and the one on right is a $3 \mathrm{D}$ surface plot. 

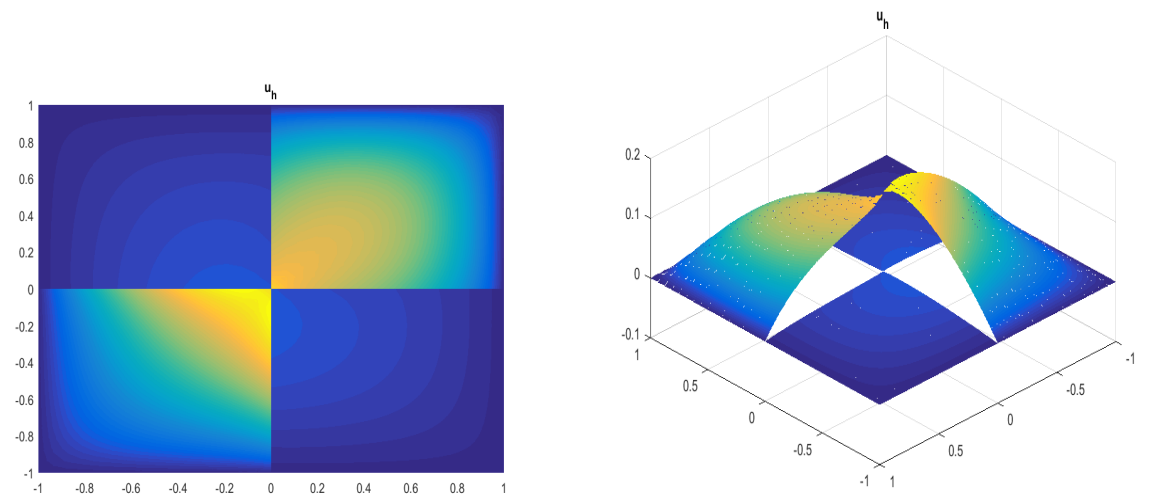

FIG. 8.3. Domain $\Omega=(-1,1) \times(-1,1)$, diffusion tensor $a=\alpha I$ with $\alpha=1$ in the first and third quadrant and $\alpha=10$ in the second and fourth quadrant, load function $f=\frac{1}{4}$, Dirichlet boundary data $g=0$, drift vector $\boldsymbol{\mu}=[1,1]$. Plot for primal variable $u_{h}$ : contour plot (left), surface plot (right).

\section{REFERENCES}

[1] I. BABŭ̌̆KA, The finite element method with Lagrange multipliers, Numer. Math., 20 (1973), pp. 179-192.

[2] L. A. Bergman, S. F. Wojtkiewicz, E. A. Johnson, and B. F. Spencer, JR., Robust numerical solution of the Fokker-Planck Equation for second order synamical systems under parametric and external white noise excitations, Fields Institute Communications, Volume 9, 1996.

[3] R. G. Bhandari And R. E. SherRer, Random vibrations in discrete nonlinear dynamic systems, J Mech Eng Sci, Vol 10, 1968, pp. 168-174.

[4] S. C. Brenner and L. R. Scott, The Mathematical Theory of Finite Element Methods, 3rd ed., Texts Appl. Math. 15, Springer, New York, 2008.

[5] F. BrezzI, On the existence, uniqueness, and approximation of saddle point problems arising from Lagrange multipliers, RAIRO, 8 (1974), pp. 129-151.

[6] E. BuRman, Error estimates for stabilized finite element methods applied to illposed problems, C. R. Acad. Sci. Paris, Ser. I 352 (2014), pp. 655-659. http://dx.doi.org/10.1016/j.crma.2014.06.008

[7] E. Burman, Stabilized finite element methods for nonsymmetric, noncoercive, and ill-possed problems. Part I: elliptic equations, SIAM J. Sci. Comput, Vol. 35, No. 6, 2013, pp. A2752A2780.

[8] E. Burman, Stabilized finite element methods for nonsymmetric, noncoercive, and ill-possed problems. Part II: hyperbolic equations, SIAM J. Sci. Comput, Vol. 36, No. 4, 2014, pp. A1911-A1936.

[9] P.G. Ciarlet, The Finite Element Method for Elliptic Problems, Classics Appl. Math. 40, SIAM, Philadelphia, 2002.

[10] L. Davis, JR., Modified Fermi mechanism for the acceleration of Cosmic Rays, Physical Review, Vol 101, Number 1, 1956, pp. 351-358.

[11] A. D. Fokker, Die mittlere Energie rotierender elektrischer Dipole im Strahlungsfeld, Ann. Phys. 348 (4. Folge 43):810-820, 1914. doi:10.1002/andp.19143480507.

[12] C. W. Gardiner, Handbook of stochastic methods, 2nd ed., Springer-Verlag, Berlin, Heidelberg, 1985.

[13] David Gilbarg and Neil S. Trudinger. Elliptic Partial Differential Equations of Second Order. Springer-Verlag, Berlin, second edition, 1983.

[14] V. Girault And P. A. Raviart, Finite Element Methods for the Navier-Stokes Equations: Theory and Algorithms, Springer-Verlag, Berlin, 1986.

[15] P. Grisvard, Elliptic Problems in Nonsmooth Domains, Classics Appl. Math. 69, SIAM, Philadelphia, 2011.

[16] P. Kumar and S. Narayana, Solution of Fokker-Planck equation by finite element and finite 
difference methods for nonlinear systems, Sadhana, Vol 31, Part 4, 2006, pp. 445-461. 2006.

[17] R. S. LANGLEy, A finite element method for the statistics of nonlinear random vibration, Journal of Sound and Vibration, 101(1), 1985, pp. 41-54.

[18] H. P. Langtangen, A general numerical solution method for Fokker-Planck equations with applications to structural reliability, Probabilistic Engineering Mechanics, 6(1), 1991, pp. $33-48$.

[19] L. Mu, J. WANG, AND X. YE, Weak Galerkin finite element methods on polytopal meshes, International Journal of Numerical Analysis and Modeling, vol. 12, pp. 31-53, 2015. arXiv:1204.3655 2 .

[20] L. Mu, J. Wang J, X. Ye, S. Zhang, $A C^{0}$-weak Galerkin finite element method for the biharmonic equation. arXiv: 1212.0250v1. Journal of Scientific Computing, Volume 59, Issue 2, 2014, pp. 473-495.

[21] A. Masud and L. A. Bergman, Application of multi-scale finite element methods to the solution of the Fokker-Planck equation, Comput. Methods Appl. Mech. Engrg. 194 (2005), pp. 1513-1526.

[22] B. T. Park And V. Petrosian, Fokker-Planck equations of stochastic acceleration: a study of numerical methods, The Astrophysical Journal Supplement Series, 103:255-267, 1996.

[23] M. Planck, Über einen Satz der statistischen Dynamik und seine Erweiterung in der Quantentheorie, Sitzungsber. Preuss. Akad. Wiss. 24, 1917.

[24] H. Risken, The Fokker-Planck equation: methods of solution and applications, 2nd Edition, Springer-Verlag, Mathematics in Science and Engineering, Vol. 60., 1989.

[25] I. Smears and E. Süli, Discontinuous Galerking finite element approximation of nondivergence form elliptic equations with Cordès coefficients, SIAM J Numer. Anal., Vol. 51, No. 4, 2013, pp. 2088-2106.

[26] B. F. Spencer and L. A. Bergman, On the numerical solutions of the Fokker-Planck equations for nonlinear stochastic systems, Nonlinear Dynamics, Vol 4, 1993, pp. 357-372.

[27] R. L. Stratonovich, Some Markov methods in the theory of stochastic processes in nonlinear dynamical systems, in: F. Moss, P. V. E. McClintock (Eds.), Noise in nonlinear dynamical systems (Vol. 1), Cambridge Univ. Press, 1989, pp. 1668.

[28] C. WANG AND J. WANG, An efficient numerical scheme for the biharmonic equation by weak Galerkin finite element methods on polygonal or polyhedral meshes, available at arXiv:1303.0927v1. Computers and Mathematics with Applications, 68 (2014), pp. 23142330. DOI:10.1016/j.camwa.2014.03.021.

[29] C. WANG AND J. WANG, A hybridized weak Galerkin finite element method for the biharmonic equation, submitted to International Journal of Numerical Analysis and Modeling, Vol. 12, 2015, pp. 302 - 317. 2015 arXiv:1402.1157v1.

[30] C. Wang And J. WANG, A primal-dual weak Galerkin finite element method for second order elliptic equations in non-divergence form, arXiv:1510.03499, Math Comp, to appear.

[31] J. WANG AND X. YE, A weak Galerkin finite element method for second-order elliptic problems, J. Comp. and Appl. Math., vol. 241, pp. 103-115, 2013. arXiv:1104.2897v1.

[32] J. WAng And X. YE, A weak Galerkin mixed finite element method for second-order elliptic problems, available at arXiv:1202.3655 1. Math. Comp., 83 (2014), pp. 2101-2126.

[33] J. WANG AND X. YE, A weak Galerkin finite element method for the Stokes equations. arXiv:1302.2707v1. Advances in Computational Mathematics, Volume 42, Issue 1, pp. 155174, 2016. DOI 10.1007/s10444-015-9415-2. 